\title{
Assessing tumor infiltrating lymphocytes in solid tumors: a practical review for pathologists and proposal for a standardized method from the International Immuno-Oncology Biomarkers Working Group:
}

\author{
Part 1: Assessing the host immune response, TILs in invasive breast carcinoma and ductal \\ carcinoma in situ, metastatic tumor deposits and areas for further research
}

A full list of authors and affiliations appears at the end of the article.

\begin{abstract}
Assessment of tumor infiltrating lymphocytes (TILs) in histopathological specimens can provide important prognostic information in diverse solid tumor types, and may also be of value in predicting response to treatments. However, implementation as a routine clinical biomarker has not yet been achieved. As successful use of immune checkpoint inhibitors and other forms of immunotherapy become a clinical reality, the need for widely applicable, accessible and reliable immuno-oncology biomarkers is clear. In Part 1 of this review we briefly discuss the host immune response to tumors and different approaches to TIL assessment. We propose a standardized methodology to assess TILs in solid tumors on H\&E sections, in both primary and metastatic settings, based on the International Immuno-Oncology Biomarker Working Group guidelines for TIL assessment in invasive breast carcinoma. A review of the literature regarding the value of TIL assessment in different solid tumor types follows in Part 2. The method we propose is reproducible, affordable, easily applied, and has demonstrated prognostic and predictive significance in invasive breast carcinoma. This standardized methodology may be used as a reference against which other methods are compared, and should be evaluated for clinical validity and utility. Standardization of TIL assessment will help to improve consistency and reproducibility in this field, enrich both the quality and quantity of comparable evidence, and help to thoroughly evaluate the utility of TILs assessment in this era of immunotherapy.
\end{abstract}

\section{Keywords}

Lymphocytes; tumor-infiltrating; Biomarkers; Cancer; Immunotherapy; Pathology

Corresponding author: Dr. Roberto Salgado, Department of Pathology, Oosterveldlaan 24, 2610 Wilrijk, Belgium, roberto@ salgado.be, Tel: ++32 4978912 18. Prof. Stephen Fox, Department of Pathology, Peter MacCallum Cancer Centre, 305 Grattan St, Melbourne, Victoria 3000, Australia, stephen.fox@ petermac.org, Tel: +61 385595464

The authors have no conflicts of interest to disclose. 


\section{Introduction}

Pathologists have long recognized the stroma, immune infiltrate, nerves and vasculature as integral parts of the tumor microenvironment, which often provide important information regarding tumor behavior, prognosis and response to treatment. It is well established that tumors are antigenic and can induce an immune response, due in part to altered protein products that may be recognized as foreign by the host immune system [1,2]. A growing body of research has shown that the extent and composition of the host immune response to the tumor has prognostic and predictive significance in many solid malignancies (reviewed in [3]). The assessment of immune infiltrate in tumors, most commonly referred to as tumor infiltrating lymphocytes (TILs), is also gaining importance in the current quest for optimal biomarkers to select patients with the highest likelihood of responding to immunotherapeutic agents. Therefore, TIL assessment has been proposed as a biomarker for inclusion in routine histopathological reporting [4,5]. Current TIL scoring systems used in research and proposed for different tumor types vary widely in detail, scope, accuracy, and time and resource requirements.

Development of prognostic and predictive biomarkers in oncology requires robust assessment of the test's analytical validity, clinical validity and clinical utility [6,7]. Evidence is accumulating to support the use of TILs scoring as a prognostic biomarker in various solid tumors and evidence for the predictive benefit of TILs is being investigated at present. Different methods of assessing TILs will have different pre-analytical, analytical and post-analytical challenges. For example, semi-quantitative H\&E based scores may suffer from low precision and poor inter-observer reproducibility if no clear guidance exists, while digital quantification of IHC stained sections may produce different results due to inaccurate measurement of the test variable without controlled calibration. Testing of the clinical validity of biomarkers involves determining the extent to which the biomarker predicts the clinical outcome of interest, that is, patient prognosis or response to treatment [7]. Assessment of the clinical validity of TILs scoring requires a standardized, reproducible method, which can be validated preferably in several independent populations. Many biomarker studies are observational, retrospective studies in which the study population is selected solely by the availability of samples [8,9]. While prospective controlled studies designed to test biomarkers are rare and unlikely to be performed on a large scale, prospective-retrospective studies may offer a comparable level of evidence [8]. These prospective-retrospective studies involve use of samples collected during a prospective randomized clinical trial, and allow high quality evaluation of the biomarker of interest provided the study design meets certain criteria and results can be replicated in an independent population [8]. Guidelines for the reporting of biomarker studies are available $[9,10]$ and should be considered when evaluating the TILs literature.

In part 1 of this review, we aim to briefly describe the host immune response to tumors and approaches used to assess this in the current context of immunotherapy. We propose a standardized methodology for TIL assessment in solid tumors, based on the International Immuno-Oncology Biomarkers Working Group guidelines for TIL assessment in invasive breast carcinoma, which may be adapted to different tumor types. We then discuss the literature and our experiences in the areas of invasive breast cancer, ductal carcinoma in situ 
(DCIS) and metastatic tumor deposits, then conclude with a discussion of open questions and areas for further research. In part 2 of this review, the literature surrounding methods of TIL assessment and the prognostic and potentially predictive significance of TILs in different solid tumors is discussed, including carcinomas of the lung, colon, upper gastrointestinal tract, head and neck, genitourinary tract, and gynecological organs, as well as mesothelioma, melanoma and primary brain tumors. Ways in which the proposed methodology can be adapted to different tumors are suggested, based on available evidence and expert opinion. Standardization of TIL assessment will allow direct comparison of different studies, highlight areas for further research, and form the basis of TIL assessment in routine histological practice.

\section{The host immune response}

Altered protein products caused by the genetic mutations in cancer cells can function as neoantigens, eliciting an immune response against a perceived "foreign" cell [2]. In addition, the inflammatory, hypoxic and often necrotic microenvironment of tumors sends concomitant danger signals to the host immune system [11]. Infiltrating immune cells can function to control tumor growth and progression, but can also help to create an immunosuppressive environment in which the tumor can thrive [12]. CD $8^{+}$cytotoxic T cells, T-helper 1 (Th1) cells producing interferon- $\gamma$, and natural killer cells are generally associated with favorable anti-tumor immune responses, along with macrophages polarized to an $\mathrm{M} 1$ phenotype and dendritic cells showing a DC1 phenotype. Immunosuppressive effects are seen with Th2 cells, M2 macrophages, DC2 dendritic cells, myeloid derived suppressor cells and $\mathrm{FOXP}^{+}$regulatory $\mathrm{T}\left(\mathrm{T}_{\text {reg }}\right)$ cells producing IL-10 and TGF $\beta$. B cells and plasma cells can also adopt either effector or regulatory phenotypes, and hence can carry positive or negative anti-tumor associations depending on contextual factors. This balance of the cellular constituents of the immune response is illustrated in Figure 1. The presence of tertiary lymphoid structures, aggregates which recapitulate the components and architecture of a lymph node, in the tumor microenvironment is correlated with better prognosis in different types of solid tumors (reviewed in [13]). The exact composition of the immune infiltrate can vary widely within and between tumors and clearly modulate the effectiveness of the anti-tumor response.

In research settings, many different methods are being used to investigate the host immune response to tumors. Many clinical studies have found significant results using an assessment of H\&E stained sections by trained pathologists, with qualitative or semi-quantitative scoring systems which vary according to tumor type [14-16]. Immunohistochemistry (IHC) allows definition of the majority of immune cell subsets that can be refined by combinations of markers, including $\mathrm{CD} 8^{+}$cytotoxic $\mathrm{T}$ cells, $\mathrm{CD} 4^{+} \mathrm{T}$ helper cells, $\mathrm{FOXP}^{+} \mathrm{T}_{\text {regs }}, \mathrm{B}$ cells, macrophages and dendritic cells. Other cell types such as myeloid derived suppressor cells require multiple cell surface markers for definition and are challenging to identify on serial IHC sections. Digital image analysis has been validated in multiple studies and can provide accurate quantitation of immune cell infiltrates in IHC stained sections [17,18]. Multiplexed fluorescent immunohistochemistry with multispectral imaging is a recent development that allows in-situ identification of different immune cell subsets on the same section, providing quantitative information on the distribution and composition of the immune infiltrate on 
formalin fixed, paraffin embedded (FFPE) tissue [19-21]. This technology requires a significant investment in initial optimization, has complex data analysis and storage requirements, and is yet to become routine. Recently, a novel approach to multiplexed IHC was described utilizing NanoString ${ }^{\circledR}$ nCounter ${ }^{\circledR}$ fluorescent barcodes to identify bound antibodies, allowing quantitation of multiple proteins in situ on an FFPE slide [22]. FFPE tissue can also be used for matrix assisted laser desorption/ionization-imaging mass spectrometry (MALDI-IMS), a proteomic technique that can identify hundreds proteins in situ without the need for specific antibodies $[23,24]$. These exciting new technologies, termed "molecular histology" combine the spatial and architectural information from traditional histology approaches with detailed molecular profiling, which is likely to be particularly relevant in describing the immune microenvironment of tumors.

Flow cytometry is a common approach to immune cell profiling and has many benefits including the characterization of immune cell subsets by multiple markers, quantitative data acquisition, wide availability and the ability to examine small subpopulations of interest [25]. However, fresh tissue is required and no information is provided on the distribution or organization of the immune infiltrate or relationship to other microenvironmental structures. Nevertheless, recent study of TILs in invasive breast carcinoma found a significant positive correlation between fresh tumor tissue analyzed by flow cytometry and IHC stained sections scored by a pathologist [26]. Messenger RNA (mRNA) profiling of tumor tissue can detect "immune gene signatures", using the level of expression of immune related genes to describe the composition and functional status of the immune infiltrate [27]. Again, no information is provided on the distribution of the infiltrate and this resource intensive technology is currently largely restricted to a research setting. These more complex methods are by their nature more difficult to implement in large, multicenter clinical trials, which ultimately are required for the validation of potential biomarkers. The costs of such detailed techniques must also be weighed against the additional information that may be derived regarding the composition and functional status of the immune infiltrate.

The host immune response to tumors is currently of great interest to oncologists and researchers following impressive early results of immune checkpoint inhibitor therapy. An important mechanism of tumor immune evasion is the expression of immune checkpoint molecules such as CTLA-4 and PD-L1, both on tumor cells and on infiltrating immune cells [28]. By blocking these signaling pathways, immune checkpoint inhibitors can re-activate the host immune system to recognize and control the tumor [28]. Clinical trials have demonstrated often durable responses in different tumor types including melanoma [29,30], urothelial carcinoma [31], Hodgkin lymphoma [32], non-small cell lung carcinoma [33-35], renal cell carcinoma [36], and squamous cell carcinoma of the head and neck [37]. However, responses within tumor types vary widely and the selection of patients likely to respond remains problematic [38]. Despite FDA approval as companion and complementary diagnostics for the use of anti-PD-1 therapy in non-small cell lung carcinoma [39,40], immunohistochemical identification of PD-L1 expression on tumor cells and/or immune cells is an imperfect biomarker [40] and a significant research effort is ongoing to identify reliable, broadly applicable and clinically valid biomarkers. T cell infiltration into tumors is critical to the success of immune checkpoint blockade [41], and tumors with high levels of infiltrating effector $\mathrm{T}$ cells, as measured by gene expression profiling, appear to show 
improved responses [42-44]. Assessment of TILs in this context is a highly active area of research and guidance for a standardized methodology is therefore timely.

\section{Proposal for a standardized methodology for scoring TILs in solid tumors, in both primary and metastatic settings}

Much research has been performed to establish the prognostic and predictive significance of TILs in different solid tumors. However, further work is needed to ensure that the valuable information that could be obtained from TILs assessment is not lost due to issues of resource commitments, methodology, or lack of standardization. It is our view that a semiquantitative H\&E based TILs assessment provides clinically relevant information in a format that is applicable to large-scale randomized clinical trials, to pre-clinical and clinical research projects and to everyday pathology practice, whether it be in high, middle, or lowincome countries. It needs to be emphasized that the sophisticated tools mentioned above are resource-intensive and may be difficult to implement in middle or low-income settings. A biomarker based on a plain H\&E stained section is affordable and accessible, and will not add to the often-restrictive costs of accessing therapy. This is an important consideration in the current era of immunotherapeutics.

Over the past few years, Roberto Salgado, Sherene Loi and Carsten Denkert have developed the International Immuno-Oncology Biomarker Working Group on Breast Cancer, with members including important clinical research groups, pathologists, clinicians and statisticians worldwide currently knowledgeable in the field of immuno-oncology biomarkers. The purpose of this Working Group is to develop, in a timely manner, standards on the assessment of immuno-oncology biomarkers to aid pathologists, clinicians and researchers in their research and daily practice. The group has, for example, developed the first International Guidelines on TIL-Assessment in Breast Cancer [14] and other guideline papers are in development. In addition to the breast cancer experts already included, academic expert groups from other fields and biomarker expert groups from industry were contacted and enthusiastically agreed to be a part of this initiative. We include worldwide representatives of known clinical research groups from expert centers across all continents (US, Europe, Australia, the Middle East and Japan) and the member list is growing.

For these papers, a panel of pathologists, medical oncologists, biostatisticians and translational researchers from different expert groups conducted a systematic review of the literature. Panel members have had experience in TIL assessment in pre-clinical research, clinical trials or are involved in translational research focused on the interactions between immunology and cancer. There are no existing guidelines on TIL assessment in solid tumors available for comparison; neither are there proficiency testing data available from international organizations. No specific funding was obtained for this project. The methodology we propose is based on the International Guidelines on TIL Assessment in Breast Cancer [14], following robust evidence from prospective retrospective phase III trials in breast cancer. Reviewed studies were not limited to randomized trials, but also included consecutive and retrospective series and in-press publications. Ways in which this methodology could be adapted to different tumor types, while remaining as standardized as 
possible, were suggested, reviewed and discussed by members of the author group expert in the particular field, to reach the consensus opinions presented here. Where strong published evidence was lacking, the panel undertook a formal expert consensus-based process by regular mail and face-to-face meetings at the San Antonio Breast Cancer Symposium 2016. Further updates and revisions are planned as more evidence becomes available and the field progresses.

We propose a method for TIL assessment that would be applicable to all solid tumors (Table 1, Figure 2). As for any proposed scoring system, this method should be evaluated for clinical validity and utility, as well as inter-observer reproducibility. This method may be used as a reference against which other systems, such as IHC-based digital quantification or mRNA profiling, are compared, to establish a sensible balance between simplicity and information yield. Individual tumor types have particular issues surrounding TILs that may require further investigation to resolve, and this method may be adapted to incorporate those particularities (see Table 2). However, most of the issues raised are common to many solid tumor types, such as the relative importance of stromal TILs and intra-tumoral TILs or the delineation of the central tumor and the invasive margin, and we feel that the method outlined in Table 1 should be able to be applied in most research and clinical settings. Pragmatic definitions of the invasive margin and central tumor areas are discussed further in part 2 of this review, but are illustrated in Figure 3 and summarized as follows: the invasive margin is defined as a $1 \mathrm{~mm}$ wide zone centered on the border of the malignant cells with the host tissue, and the central tumor is defined as the central tumor tissue surrounded by this zone. Tutorials illustrating TIL assessment in different tumor types and reference scoring sheets illustrating densities of stromal TILs are available as supplementary material online. Having a reference method to score TILs will help to ensure that potential future studies investigating TILs in each tumor type can be compared. Many potentially useful biomarkers such as the Ki67 proliferative index in breast cancer suffer from a plethora of noncomparable methods, which affects the level of evidence that can be obtained and prevents uniform clinical implementation. International efforts at standardization are also being explored for this important biomarker [45].

\section{TILs in invasive breast carcinoma}

The wide variation in the immune infiltrate seen in breast carcinomas is well known to pathologists, with an early description of medullary breast carcinoma by Moore and Foote in 1949 highlighting the lymphocytic infiltrate as a key diagnostic criterion and linking it to good prognosis [46]. This lymphocytic infiltrate was suggested to represent a form of host resistance to the tumor, however conflicting results regarding the prognostic significance of TILs were found in subsequent studies [47-50]. In addition, it was recognized that a prominent lymphocytic infiltrate may be found in tumors not meeting the other strict criteria for medullary breast carcinoma [50]. Most breast carcinomas show some degree of lymphocytic infiltrate, with higher rates of "lymphocyte predominant breast carcinoma", variably defined as $>50 \%$ or $>60 \%$ of tumor area occupied by TILs, seen in triple negative breast carcinoma (20\%) and HER2 positive breast carcinoma (16\%) compared with estrogen receptor positive breast carcinoma (6\%) [51]. Recently, through large-scale international efforts, evidence has accumulated to support the prognostic and potential predictive impact 
of TILs, both in triple negative and HER2 positive cancers. The clinical utility of assessing TILs in breast cancer relates to risk prediction models, adjuvant and neoadjuvant chemotherapy decisions, and the growing potential of immunotherapy [52].

Several large systematic reviews and meta-analyses have confirmed that high levels of TILs are associated with better disease free survival and overall survival only in triple negative and HER 2 positive subtypes, with no significant benefit seen in estrogen receptor positive breast carcinoma [53-55]. Pooled hazard ratios for overall survival with variably defined "high TILs" ranged from 0.71 to 0.88 [53-55]. A recent pooled analysis of five large clinical trials of anthracycline chemotherapy in triple negative breast carcinoma presented similar results, with an adjusted relative risk reduction of $12 \%$ for disease free and overall survival for every $10 \%$ increment in sTILs [56]. Both intra-tumoral TILs (iTILs) and stromal TILs (sTILs) gave similar results when separately assessed [53,55-57], however iTILs did not appear to add prognostic information to sTILs in a multivariate model [56]. The majority of these data come from large prospective-retrospective studies, associated with randomized clinical trials [14,56,58-65], however smaller retrospective studies were also included in the meta-analyses. Whilst many of the included studies follow the TIL scoring method used by Denkert in 2010 [60], there is variation with some studies using H\&E assessment $[14,58,59,61,62,65]$, some using immunohistochemistry [63,64], and others using mRNA expression profiling [63]. Conclusions from some recent meta-analyses in this field [66,67] indicate that care must be taken when interpreting the results of studies of prognostic biomarkers. For example, it is suggested that FOXP $3^{+} \mathrm{T}_{\text {regs }}$ correlate with poor prognosis in breast cancer $[66,67]$, however $\mathrm{T}_{\text {reg }}$ levels also correlate with other poor prognostic factors such as estrogen receptor-negativity, HER2-positivity, lymph node metastasis and high histological grade [68-71]. Within the estrogen receptor-negative subgroup, high levels of $\mathrm{FOXP}^{+} \mathrm{T}_{\text {regs }}$ may actually correlate with improved prognosis as markers of an active immune response $[68,70]$. Both primary studies and meta-analyses should report the results of multivariate analyses to confirm the prognostic value of the biomarker in question, independent of other known prognostic factors [10]. Despite these inconsistencies, there appears to be a robust prognostic benefit of a high lymphocytic infiltrate in triple negative and HER2 positive breast cancer, irrespective of the exact methodology or scoring system used.

TILs may also have value in predicting benefit from neoadjuvant chemotherapy in subsets of breast cancer patients. Both cytotoxic chemotherapy and endocrine therapy may partly function through immunomodulatory effects. Anthracyclines such as doxorubicin induce immunogenic cell death and depend on adaptive and innate immunity for therapeutic efficacy [72,73], and it has been suggested that treatment with the aromatase inhibitor letrozole, with or without cytotoxic chemotherapy, may counteract some of the immunosuppressive effects of estrogen signaling through reduction in tumoral $\mathrm{T}_{\text {regs }}$ [74]. Higher levels of TILs, both $\mathrm{CD} 8^{+} \mathrm{T}$ cells and $\mathrm{FOXP}^{+} \mathrm{T}_{\text {regs }}$, in pre-treatment biopsies correlated with higher rates of pathological complete response to neoadjuvant chemotherapy in triple negative and HER2 positive breast carcinoma [14,60-63,75]. A meta-analysis of six large clinical trials found high sTILs were a strong predictive maker for response to neoadjuvant chemotherapy in triple negative, HER2 positive and luminal subtypes, however an overall survival benefit was not seen in the luminal cancers [76]. This association is 
strongest for lymphocyte predominant breast carcinoma $[60,62,76]$ and appears to be independent of the type of chemotherapy used [52,75]. Indeed, in the NeoALLTO trial of neoadjuvant chemotherapy in HER2 positive breast carcinoma, high levels of TILs at diagnosis provided prognostic information independent of pathological complete response [14]. Early data suggest that iTILs may be of particular importance in this setting, with iTILs but not sTILs significantly associated with pathological complete response in triple negative breast carcinoma [77]. In addition, the presence of TILs in post-treatment specimens with residual disease appears to confer a better prognosis than those showing an absence of TILs $[78,79]$. Guidelines from the International Immuno-Oncology Biomarker Working Group for assessing TILs in the residual disease setting will soon be available.

Recently published consensus guidelines provide detailed instructions and illustrations to aid pathologists in the assessment of TILs in breast cancer [14]. These guidelines were developed at a meeting of major international breast cancer research teams (the International TILs Working Group, now known as the International Immuno-Oncology Biomarker Working Group) in 2013, where it was recognized that standardized methodology and scoring systems are critical to the integration of information about TILs into future research and eventually into routine diagnostic practice [14]. The proposed methodology is based on that used by Denkert et al in 2010 [60], and requires assessment of only one H\&E slide per specimen, as detailed in Table 3. Briefly, TILs are reported as an overall percentage of the stromal area within the borders of the invasive tumor that is covered by mononuclear immune cells. Care is taken to exclude any lymphocytic infiltrate around normal lobules, in the previous biopsy site or in areas of diathermy or crush artifact. It is stressed that no recommendation can currently be made for a clinically relevant cut off, and that TILs should be reported as a continuous variable, in as much detail as is comfortable to the reporting pathologist [14]. A tutorial outlining this methodology to score TILs in invasive breast cancer is available online in Supplementary File 1, and a reference scoring sheet to illustrate different levels of sTILs in available in Supplementary File 2.

In this method, only stromal TILs are assessed. Similar prognostic associations have been identified with both sTILs and iTILs, defined as TILs directly interacting with carcinoma cells with no intervening stroma $[65,78]$. The tumor stroma and the immune cells within it are integral parts of the tumor microenvironment, thus "stromal TILs" within the borders of the invasive tumor can be considered true tumor infiltrating lymphocytes. It was felt that the assessment of intra-tumoral TILs in addition to stromal TILs would be unlikely to add value currently since they can be challenging to detect on H\&E, are present in fewer cases and are dependent on the size and distribution of tumor nests [14]. However, due to the difficulties recognizing iTILs on an $\mathrm{H} \& \mathrm{E}$, their importance may well be underestimated. Efforts are ongoing to identify and characterize iTILs using IHC and/or digital analysis tools, enabling a more accurate assessment of the potential clinical significance of TILs in this compartment. For example, CD103 is a marker of a subset of CD8 ${ }^{+}$TILs that are most prevalent amongst intra-tumoral TILs and may reflect $\mathrm{CD} 8^{+} \mathrm{T}$ cells that have been engaged in an adaptive immune response [80]. Whereas TIL assessment on H\&E was of borderline significance in a small cohort of basal-like invasive breast tumors, dual $\mathrm{CD} 103^{+} \mathrm{CD} 8^{+} \mathrm{TIL}$ status was strongly prognostic for relapse-free and overall survival [81]. The intensity and distribution of TILs in breast cancers is often heterogeneous $[82,83]$ and it is recommended 
to give an overall assessment rather than focusing on hotspots of intense infiltration, based on a lack of data demonstrating any clinical significance of this heterogeneity [14]. Recently, Mani et al demonstrated more heterogeneity within a single biopsy than between biopsies or regions from a given breast cancer, suggesting that the average lymphocyte score from a single biopsy may be representative of the whole tumor [82], supported by another recent study showing high correlation between TILs assessed on a pre-operative core biopsy and those assessed on the resection specimen [84]. Interestingly, despite the lack of functional information gained from assessing lymphocytes on H\&E only, studies using this method have shown similar prognostic results to those separating lymphocyte subsets by immunohistochemistry [14]. In addition, assessment of TILs on H\&E stained sections gave similar predictive information to mRNA expression profiling [61]. It is emphasized that the method proposed leaves a number of open questions requiring further investigation, discussed further below. Refinement of the method is ongoing and updated guidelines will be issued as new evidence becomes available.

Large international reproducibility ring studies by the International Immuno-Oncology Biomarker Working Group have recently been reported [52]. Based on the 2014 guidelines, an initial web-based assessment of 120 slides by 32 pathologists yielded good agreement with an intra-class correlation coefficient (ICC) of 0.70 (95\% CI 0.62-0.78). Although high, this ICC did not meet the pre-specified endpoint, and therefore a second round was undertaken, using specifically developed software, which provided visual reference ranges and integrated feedback. ICC improved to 0.89 (95\% CI 0.85-0.92) and it was suggested that visual reference tools such as the software used in this study might be of value both in research and in clinical practice [14,52]. A recent study has shown similarly high interobserver and intra-observer ICC for scoring TILs in invasive breast carcinoma, however it was noted that agreement for the iTILs scores was lower than for the sTILs scores [84]. There were no significant differences between the inter- and intra-observer ICC values when scoring H\&E sections or IHC stained sections, and concordance between H\&E and IHC scores was high (ICC 0.75 for iTIL and ICC 0.84 for sTIL) [84]. Further reproducibility studies such as these are important to thoroughly validate TILs as a biomarker, compare different methodologies, and to work towards a consensus on the degree of reproducibility required for TIL assessment and for biomarkers more generally.

\section{TILs in ductal carcinoma in situ}

In comparison to invasive breast carcinoma, less is known about the significance of the immune infiltrate associated with DCIS. Whilst features of the stromal microenvironment of DCIS, such as periductal myxoid change, angiogenesis and stromal proteins, are acknowledged as being important in determining disease outcome $[85,86]$, the immune infiltrate surrounding ducts involved by DCIS has received relatively little attention. Early studies do however suggest that TILs are associated with certain clinicopathological features and disease progression, but may not have prognostic significance in this setting.

Like invasive breast carcinoma, some degree of lymphocytic infiltration is often present within or surrounding ducts involved by DCIS $[87,88]$. Pruneri et al have recently reported on the prevalence and clinical relevance of TILs in DCIS, in a large series with substantial 
length of follow up [88]. sTILs were significantly associated with DCIS grade, patient age, and the presence of comedonecrosis, and were most common in the HER2 positive subtype [88]. No association was observed with ipsilateral recurrence, whether in situ or invasive [88]. In this study, sTILs were scored using a method based on the International ImmunoOncology Biomarker Working Group guidelines for invasive breast carcinoma [14], with some modifications specific to the DCIS setting (Table 2). A reference scoring sheet for DCIS developed by Pruneri et al is available in Supplementary File 3. This method was highly reproducible with an ICC of 0.96 (95\% CI 0.95-0.97) [88]. Thompson et al performed a semi-quantitative H\&E assessment of TILs on tissue microarray sections, followed by a comprehensive immunohistochemical examination of the lymphocyte subsets involved [87]. No significant associations were found between the H\&E based TILs score and other clinicopathological features such as hormonal status, age, or concurrent invasive breast carcinoma, however small numbers precluded complete assessment [87]. Campbell et al used both manual H\&E TIL counts and digital image analysis of IHC stained sections to comprehensively study the immune microenvironment of DCIS [89]. High-grade DCIS cases showed higher overall TILs, CD4 ${ }^{+} \mathrm{T}$ cells, CD20 ${ }^{+} \mathrm{B}$ cells, FOXP3 ${ }^{+} \mathrm{T}_{\text {regs }}$, and $\mathrm{CD} 68^{+}$ macrophages than low or intermediate grade DCIS cases [89]. An exploratory classification system identified $\mathrm{CD} 8^{+} \mathrm{HLADR}^{+} \mathrm{T}$ cells, $\mathrm{CD} 8^{+} \mathrm{HLADR}^{-} \mathrm{T}$ cells, and $\mathrm{CD} 115^{+}$macrophages to be associated with recurrence [89]. These findings suggest that specific TIL subsets may be of importance in DCIS, and these subsets cannot be differentiated on H\&E stained sections. Further research is required to evaluate this potential effect of TIL subsets on recurrence in DCIS.

The host immune response to tumors impacts disease progression [12], and this may be identifiable in pre-invasive lesions such as DCIS. Based on immunohistochemical quantitation, Hussein et al described an increase in stromal lymphocyte subsets through progression from proliferative breast disease and DCIS to invasive carcinoma [90]. Bates et al reported that the density of FOXP3 ${ }^{+} \mathrm{T}_{\text {regs }}$ in DCIS was higher than that seen in normal breast but less than in invasive carcinoma [71], with similar findings reported in 2013 by Lal et al [91]. DCIS with higher $\mathrm{T}_{\text {reg }}$ levels had a higher risk of recurrence [71]. Interestingly, similar findings have also been demonstrated in pancreatic lesions, with higher numbers of $\mathrm{T}_{\text {regs }}$ found in the stroma of invasive adenocarcinomas than around pre-malignant lesions, which showed a higher infiltrate than non-neoplastic lesions [92]. Opposite findings were noted for $\mathrm{CD}^{+}$cytotoxic $\mathrm{T}$ cells, which decreased with malignant progression [92]. Higher levels of $\mathrm{T}_{\text {regs }}$ may reflect evasion of the host immune response as the tumor progresses. Challenges remain in assessing TILs in pre-invasive lesions, including definition of the tumor area and delineation of reactive and "bystander" lymphocytes. Pruneri et al defined the tumor area as the specialized intra-lobular stroma surrounding the ducts involved by DCIS (within two high-power microscopic fields) [88]. The stroma surrounding ducts involved by DCIS is known to be altered relative to normal breast intra-lobular stroma $[85,86]$, however the functional significance of reactive TILs may be diluted by pre-existing lymphocytes in this region. Further study of pre-invasive lesions such as DCIS may provide insights into the process of immunoediting and immune evasion as invasive malignancies develop and progress. 


\section{TILs in the metastatic setting}

The immune response to metastatic tumor deposits as well as the primary tumor is of clear importance to immunotherapies, which are largely being studied and approved in advanced settings. Important differences in the immune infiltrate may exist between the primary tumor and metastatic deposits, which may reflect changes in the immune response to the tumor over time or in specific anatomical locations. In small studies of paired primary and metastatic breast carcinomas, lower levels of TILs were observed in the metastasis [93-95], a finding also demonstrated in renal cell carcinoma [96] and non-small cell lung carcinoma [97], supporting the concepts of immunoediting and immune evasion. In addition, Baine et al demonstrated a lower CD8/FOXP3 ratio in metastatic deposits of renal cell carcinoma than in the primary tumor, suggesting that the TILs in the metastasis may be more polarized towards an immunosuppressive phenotype [96]. The immune response to metastases may be site dependent; both in melanoma [98] and in HER2 positive breast carcinoma [99], metastases in the lung contained the highest numbers of TILs, although an even distribution of TILs between metastatic sites was found in renal cell carcinoma [96]. Despite the immune-privileged status of the central nervous system [100], brain metastases often demonstrate an immune infiltrate, which may be associated with improved overall survival [101,102]. As discussed in part 2 of this review, TILs in melanoma metastases have been shown to have similar prognostic importance to those in the primary lesion [103-106]. TILs in liver and lung metastases of colorectal carcinoma also show prognostic significance [107109]. An interesting observation comparing primary breast tumors and later in-breast relapses, based on a very small cohort, is that higher levels of TILs may be associated with true recurrence type relapses while new primary type relapses show lower TIL levels [110]. This is consistent with the notion that new primaries can only develop through immune evasion in the face of a primed immune system whereas true recurrences develop despite the immune response. Early data suggest an interesting interaction between TILs in metastatic breast cancer and tumor phenotype, namely high TILs in the metastatic or recurrent lesion were associated with improved prognosis in triple negative breast carcinoma but with worse prognosis in HER2 positive breast carcinoma [111]. Importantly, higher numbers of TILs at the invasive margin of melanoma metastases correlated with improved response to immune checkpoint inhibition [112].

Evaluating TILs in the most recent tumor sample available, including metastatic deposits, may be more relevant in describing the current immunological status of the patient than relying solely on the primary tumor. This practice is advocated in the assessment of other oncological biomarkers such as epidermal growth factor receptor mutation status in nonsmall cell lung carcinoma [113]. The standardized methodology we propose (Table 1) should be able to be easily adapted to the metastatic setting, as metastatic tumor deposits share many histopathological features with primary tumors. A tutorial illustrating application of this method to breast carcinoma metastasis is available online in Supplementary File 4 . The consensus method previously reported for invasive breast carcinoma has been successfully used to investigate the clinical relevance of TILs in breast cancer metastases in a subset of a large prospective-retrospective study [99]. Evaluating TILs in metastatic deposits in lymph nodes is complicated by the presence of a pre-existing 
lymphoid stroma; this issue is discussed further below. As in primary tumors, our proposed method should be thoroughly validated and shown to be reproducible in metastatic lesions. This will allow for valid comparison between primary and metastatic tumors, between different tumor types or between different metastatic sites, which may be of particular relevance in the current era of immunotherapy.

\section{Areas of uncertainty and open questions for further investigation}

Despite the evidence that is accumulating to support the prognostic and potential predictive value of TILs assessment, a number of areas of uncertainty remain. These open questions encompass a range of practical issues encountered by pathologists when scoring TILs, as well as areas of incomplete understanding in the fundamentals of tumor immunology. Assessing TILs in metastatic tumor deposits is clearly an important area for future study and early evidence suggests that assessing TILs in lymph nodes can demonstrate prognostic value $[99,103,114]$, despite the obvious difficulties in distinguishing lymphocytes truly related to the tumor from those pre-existing "bystander" lymphocytes populating the node. Tumor draining lymph nodes have important immunological and structural differences from nodes unrelated to the tumor, even before the arrival of metastatic tumor cells [115]. In addition, pre-clinical evidence suggests that systemic immunity is required for tumor control and eradication [116], not only an effective local immune microenvironment. In order to further investigate this question and acknowledging the lack of supporting evidence, we propose a pragmatic method for scoring TILs in lymph node metastases on H\&E sections illustrated in Figure 4. Metastatic tumor deposits often induce a desmoplastic stroma within the lymph node, in which case the sTILs may be scored within this reactive stroma (Figure 4, panels A-D), and the iTILs scored as those lymphocytes within the tumor nests, analogous to the primary lesion. In cases where the metastatic tumor deposit does not induce a desmoplastic stroma (Figure 4, panels E and F), we suggest limiting the evaluation to iTILs, that is, lymphocytes infiltrating and disrupting tumor cells nests. The pre-existing background lymphoid stroma should be excluded from the assessment. This method would be most applicable where the full lymph node is available for assessment. TILs may not be able to be reliably scored using this proposed method in core biopsies of involved nodes, particularly in the absence of a desmoplastic stroma. Scoring iTILs on H\&E sections is admittedly challenging, and this may be an area in which inter-observer reproducibility requires particular attention. These methods are suggested as preliminary guidelines that should be validated for reproducibility and clinical validity.

How best to describe regional heterogeneity, variation between multifocal tumors, or biphasic lesions with clear morphological differences is also not yet well defined. An average whole section assessment may better capture any heterogeneity than a hotspot or random sampling methods, however further evidence is required to rigorously compare these approaches. While core biopsies may be used in the neoadjuvant setting, whole sections are preferred. Evidence to support any recommendations on the minimum amount of tumor tissue that reliably represents the immune microenvironment of the whole tumor is not yet available. Whether TILs could potentially be assessed on cytology specimens is also a controversial but important area, particularly in the setting of lung carcinoma in which these are often the only tumor samples available. It is also uncertain how to assess mixed 
inflammatory infiltrates, for example neutrophilic, eosinophilic and histiocytic infiltrates not related to necrosis, or how to approach lymphoid infiltrates in adjacent normal tissue or adjacent non-invasive neoplastic lesions.

It must be emphasized that the margin between tumor and normal tissue, the "invasive margin", as we have defined it, may represent different growth patterns and biologies in both primary and metastatic settings. This margin may represent an infiltrative front, but could also represent a pushing front, or a mixture of the two. Currently, research is ongoing to determine whether the general immunological and biological context, and TIL infiltration patterns in particular, have different clinical meanings associated with specific growth patterns at the tumor margin. Our definition of the invasive margin, based on that proposed by Galon and colleagues [117], is a pragmatic one, which we hope will be of value in standardizing future research in this clearly important area of the interface between the tumor and the host.

As further study is undertaken and evidence becomes available, these (and other) issues may be resolved and incorporated into future guidelines. The methods we propose should be considered a tool for further investigation, to be adopted, rejected or improved upon as the field progresses.

\section{Discussion}

It is clear that the host immune response to cancer can alter tumor biology and response to treatment, a feature common to many different solid tumors, as discussed further in part 2 of this review. Many questions regarding the origin, determinants and clinical relevance of the immune response in cancer remain, and limitations to our proposed method are acknowledged. Importantly, no information regarding the immune cell subsets or functional status is obtained from assessment of an H\&E section. The balance of immune cells and soluble factors providing effective anti-tumor responses to those with immunosuppressive effects may be better described with more sophisticated techniques such as gene expression profiling [27]. Clearly not all mononuclear immune cells are $\mathrm{T}$ cells, and the relative proportions of macrophages, dendritic cells, myeloid derived suppressor cells and plasma cells in the immune infiltrate may also provide important information [3]. TILs may be exhausted or rendered inactive through immune checkpoint pathways such as PD-1:PD-L1 signaling, or lack of immune stimulatory pathways such as OX-40:OX-40L signaling. Expression of these checkpoint molecules is induced by a once-active immune response $[28,118]$, which may be reactivated through immunotherapy, chemotherapy or radiotherapy. High levels of TILs as defined on H\&E may not correspond to high levels of active antitumor TILs and this functional suppression may explain, at least in part, why some patients with high levels of TILs do not show improved prognosis. It is not yet established whether an $H \& E$ based assessment can provide sufficient information for clinical decision making in the context of immunotherapy. However, we feel that this prospect should be thoroughly investigated and not discarded prematurely due to the perceived simplicity of this approach.

The organization and distribution of the immune response may not be fully described by obtaining an average TILs score across the section. The importance of tertiary lymphoid 
structures, which represent an organized immune infiltrate with all the features of a lymph node to facilitate antigen presentation, lymphocyte education and effector cell proliferation, has been described in different tumor types [13]. A recent study suggests that IHC is required to reliably identify tertiary lymphoid structures [84], however consensus on the most appropriate marker or combination of markers for this task is not yet apparent. Whether further descriptive analysis of the immune infiltrate in tumors, incorporating features such as the density and distribution of TILs, organization into tertiary lymphoid structures, or the presence of hotspots, adds prognostic information to an overall TILs assessment remains to be determined.

Why some tumors have high levels of TILs and others do not is an ongoing area of fascinating research. It has been hypothesized that a higher mutational burden will correspond to a greater potential for neoantigen formation and a higher immune infiltrate [119]. The tumor types with high average mutation load such as melanoma and non-small cell lung carcinoma, as well as the most highly mutated individual tumors within these types, appear to respond better to immunotherapy [120,121], however the mutational load does not necessarily correlate with the expression of immune-related genes in the tumor or the effector T cell infiltrate [122-124]. Specific mutational signatures such as homologous recombination pathway defects [125], POLE mutations, or microsatellite instability may prove to be important. Alternatively, activation of specific oncogenic pathways in tumor cells, such as WNT/beta-catenin, has been implicated in preventing immune cell infiltration $[123,126]$. Access of the immune cells to the tumor via the blood vessels and tumor stroma is also a critical consideration [127]. It is clear that a standardized approach to scoring TILs will be of benefit when investigating the origin and determinants of the immune infiltrate.

It is hoped that use of a standardized methodology for TILs assessment as a reference standard will help to resolve many issues associated with interpreting future studies.

Comparison of, for example, the value of a Th1 gene expression profile with that of a simple $\mathrm{H} \& \mathrm{E}$ assessment, or the effect of mutational load versus mutational signature, will be informative to pre-clinical and clinical research. As evidence accumulates for the clinical utility of TILs as a prognostic and predictive biomarker, and as the search for reliable immunotherapy biomarkers continues, pathologists' role in assessing and quantifying immune infiltrates will increase. Technologies will advance, and detailed in situ assessment of the functional status of the immune infiltrate may soon be possible in an inexpensive and widely available manner. However, it is imperative that we develop a pragmatic methodology of generating reliable data on immune infiltrates, that can be applied in large scale clinical trials and in daily histopathology practice, even in resource poor settings. The method we propose (Table 1) has shown high reproducibility in the context of invasive breast carcinoma [52], and should be able to be applied, with modifications as required, to all solid tumor types. These pragmatic recommendations may form the basis for further definitive guidelines for different solid tumors as more evidence becomes available. It is hoped that a standardized methodology will be of value to clinicians, researchers and practicing pathologists, to develop relevant cut-offs and conclusively demonstrate the clinical utility of this simple biomarker. 


\section{Supplementary Material}

Refer to Web version on PubMed Central for supplementary material.

\section{Authors}

Shona Hendry ${ }^{1,2}$, Roberto Salgado ${ }^{3,4}$, Thomas Gevaert ${ }^{5,6}$, Prudence A. Russell7,8, Tom Johnn,10,11, Bibhusal Thapa ${ }^{10,12}$, Michael Christie ${ }^{13}$, Koen van de Vijver ${ }^{14}, \mathrm{M}$. Valeria Estrada ${ }^{15}$, Paula I. Gonzalez-Ericsson ${ }^{16}$, Melinda Sanders ${ }^{17}$, Benjamin Solomon ${ }^{18}$, Cinzia Solinas ${ }^{19}$, Gert GGM Van den Eynden ${ }^{19,20}$, Yves Allory 21,22,23, Matthias Preusser ${ }^{24}$, Johannes Hainfellner ${ }^{25}$, Giancarlo Pruneri ${ }^{26,27}$, Andrea Vingiani26,27, Sandra Demaria ${ }^{28,29}$, Fraser Symmans ${ }^{30}$, Paolo Nuciforo ${ }^{31}$, Laura Comerma $^{31}$, E. Aubrey Thompson ${ }^{32}$, Sunil Lakhani ${ }^{33,34}$, Seong-Rim Kim ${ }^{35}$, Stuart Schnitt ${ }^{36,37}$, Cecile Colpaert ${ }^{38}$, Christos Sotiriou ${ }^{39}$, Stefan J. Scherer ${ }^{40}$, Michail Ignatiadis ${ }^{39}$, Sunil Badve ${ }^{41}$, Robert H. Pierce ${ }^{42}$, Giuseppe Viale ${ }^{43}$, Nicolas Sirtaine $^{44}$, Frederique Penault-Llorca ${ }^{45,46}$, Tomohagu Sugie ${ }^{47}$, Susan Fineberg ${ }^{48,49}$, Soonmyung Paik ${ }^{35,50}$, Ashok Srinivasan ${ }^{35}$, Andrea Richardson ${ }^{37,51,52}$, Yihong Wang ${ }^{53,54}$, Ewa Chmielik ${ }^{55,56}$, Jane Brock ${ }^{37,51}$, Douglas B. Johnson ${ }^{57,58}$, Justin Balk0 ${ }^{57,58}$, Stephan Wienert ${ }^{59,60}$, Veerle Bossuyt ${ }^{61}$, Stefan Michiels ${ }^{62}$, Nils Ternes $^{62}$, Nicole Burchardi63, Stephen J. Luen²,18, Peter Savas ${ }^{2,18}$, Frederick Klauschen ${ }^{59}$, Peter H. Watson ${ }^{64,65}$, Brad H. Nelson $65,66,67$, Carmen Criscitiello ${ }^{26}$, Sandra O'Toole ${ }^{68,69}$, Denis Larsimont ${ }^{44}$, Roland de Wind ${ }^{44}$, Giuseppe Curigliano ${ }^{26}$, Fabrice André ${ }^{70,71}$, Magali Lacroix-Triki ${ }^{70}$, Mark van de Vijver ${ }^{72}$, Federico Rojo ${ }^{73}$, Giuseppe Floris $^{74}$, Shahinaz Bedri ${ }^{75}$, Joseph Sparano ${ }^{76}$, David Rimm ${ }^{61}$, Torsten Nielsen ${ }^{77}$, Zuzana Kos ${ }^{78}$, Stephen Hewitt ${ }^{79}$, Baljit Singh ${ }^{80}$, Gelareh Farshid ${ }^{81,82}$, Sibylle Loibl ${ }^{63}$, Kimberly H. Allison ${ }^{83}$, Nadine Tung ${ }^{84}$, Sylvia Adams ${ }^{28,29}$, Karen Willard-Gallo ${ }^{19}$, Hugo M. Horlings ${ }^{85}$, Leena Gandhi29,86, Andre Moreira ${ }^{87}$, Fred Hirsch ${ }^{88}$, Maria Vittoria Dieci ${ }^{89,90}$, Maria Urbanowicz ${ }^{91}$, Iva Brcic ${ }^{92}$, Konstanty Korski $^{93}$, Fabien Gaire ${ }^{93}$, Hartmut Koeppen ${ }^{94}$, Amy Lo ${ }^{94,95}$, Jennifer Giltnane ${ }^{94}$, James Ziai ${ }^{94}$, Marlon C. Rebelatto ${ }^{96}$, Keith E. Steele ${ }^{96}$, Jiping Zha ${ }^{96}$, Kenneth Emancipator ${ }^{97}$, Jonathan W. Juco ${ }^{97}$, Carsten Denkert ${ }^{59}$, Jorge Reis-Filho ${ }^{98}$, Sherene Loi ${ }^{18}$, and Stephen B. Fox ${ }^{1,2}$

\section{Affiliations}

${ }^{1}$ Department of Pathology, Peter MacCallum Cancer Centre, Melbourne, Australia ${ }^{2}$ The Sir Peter MacCallum Department of Oncology, University of Melbourne, Parkville, Australia ${ }^{3}$ Breast Cancer Translational Research Laboratory/Breast International Group, Institut Jules Bordet, Brussels, Belgium ${ }^{4}$ Department of Pathology and TCRU, GZA, Antwerp, Belgium ${ }^{5}$ Department of Development and Regeneration, Laboratory of Experimental Urology, KU Leuven, Leuven, Belgium ${ }^{6}$ Department of Pathology, AZ Klina, Brasschaat, Belgium ${ }^{7}$ Department of Anatomical Pathology, St Vincent's Hospital Melbourne, Fitzroy, Australia ${ }^{8}$ Department of Pathology, University of Melbourne, Parkville, Australia ${ }^{9}$ Department of Medical Oncology, Austin Health, Heidelberg, Australia ${ }^{10}$ Olivia Newton-John Cancer Research Institute, Heidelberg, Australia ${ }^{11}$ School of Cancer Medicine, La Trobe University, Bundoora, Australia ${ }^{12}$ Department of Medicine, University of 
Melbourne, Parkville, Australia ${ }^{13}$ Department of Anatomical Pathology, Royal Melbourne Hospital, Parkville, Australia ${ }^{14}$ Divisions of Diagnostic Oncology \& Molecular Pathology, Netherlands Cancer Institute-Antoni van Leeuwenhoek, Amsterdam, The Netherlands ${ }^{15}$ Department of Pathology, School of Medicine, University of California, San Diego, USA ${ }^{16}$ Breast Cancer Research Program, Vanderbilt Ingram Cancer Center, Vanderbilt University, Nashville, USA ${ }^{17}$ Department of Pathology, Microbiology and Immunology, Vanderbilt University Medical Center, Nashville, USA ${ }^{18}$ Department of Medical Oncology, Peter MacCallum Cancer Centre, Melbourne, Australia ${ }^{19}$ Molecular Immunology Unit, Institut Jules Bordet, Université Libre de Bruxelles, Brussels, Belgium ${ }^{20}$ Department of Pathology, GZA Ziekenhuizen, Antwerp, Belgium ${ }^{21}$ Université Paris-Est, Créteil, France ${ }^{22}$ INSERM, UMR 955, Créteil, France ${ }^{23}$ Département de pathologie, APHP, Hôpital Henri-Mondor, Créteil, France ${ }^{24}$ Department of Medicine, Clinical Division of Oncology, Comprehensive Cancer Centre Vienna, Medical University of Vienna, Vienna, Austria ${ }^{25}$ Institute of Neurology, Comprehensive Cancer Centre Vienna, Medical University of Vienna, Vienna, Austria ${ }^{26}$ European Institute of Oncology, Milan, Italy ${ }^{27}$ University of Milan, School of Medicine, Milan, Italy ${ }^{28} \mathrm{New}$ York University Medical School, New York, USA ${ }^{29}$ Perlmutter Cancer Center, New York, USA ${ }^{30}$ Department of Pathology, University of Texas M.D. Anderson Cancer Center, Houston, USA ${ }^{31}$ Molecular Oncology Group, Vall d'Hebron Institute of Oncology, Barcelona, Spain ${ }^{32}$ Department of Cancer Biology, Mayo Clinic, Jacksonville, USA ${ }^{33}$ Centre for Clinical Research and School of Medicine, The University of Queensland, Brisbane, Australia ${ }^{34}$ Pathology Queensland, Royal Brisbane and Women's Hospital, Brisbane, Australia ${ }^{35}$ National Surgical Adjuvant Breast and Bowel Project Operations Center/NRG Oncology, Pittsburgh, Pennsylvania ${ }^{36}$ Cancer Research Institute and Department of Pathology, Beth Israel Deaconess Cancer Center, Boston, USA ${ }^{37}$ Harvard Medical School, Boston, USA ${ }^{38}$ Department of Pathology, GZA Ziekenhuizen, Sint-Augustinus, Wilrijk, Belgium ${ }^{39}$ Department of Medical Oncology, Institut Jules Bordet, Université Libre de Bruxelles, Brussels, Belgium ${ }^{40}$ Academic Medical Innovation, Novartis Pharmaceuticals Corporation, East Hanover, USA ${ }^{41}$ Department of Pathology and Laboratory Medicine, Indiana University, Indianapolis, USA ${ }^{42}$ Cancer Immunotherapy Trials Network, Central Laboratory and Program in Immunology, Fred Hutchinson Cancer Research Center, Seattle, USA ${ }^{43}$ Department of Pathology, Istituto Europeo di Oncologia, University of Milan, Milan, Italy ${ }^{44}$ Department of Pathology, Institut Jules Bordet, Université Libre de Bruxelles, Brussels, Belgium ${ }^{45}$ Department of Surgical Pathology and Biopathology, Jean Perrin Comprehensive Cancer Centre, Clermont-Ferrand, France ${ }^{46}$ University of Auvergne UMR1240, Clermont-Ferrand, France ${ }^{47}$ Department of Surgery, Kansai Medical School, Hirakata, Japan ${ }^{48}$ Montefiore Medical Center, Bronx, New York, USA ${ }^{49}$ The Albert Einstein College of Medicine, Bronx, New York, USA ${ }^{50}$ Severance Biomedical Science Institute and Department of Medical Oncology, Yonsei University College of Medicine, Seoul, South Korea ${ }^{51}$ Department of Pathology, Brigham and Women's Hospital, Boston, USA ${ }^{52}$ Department of Cancer Biology, Dana Farber Cancer Institute, Boston, USA 
${ }^{53}$ Department of Pathology and Laboratory Medicine, Rhode Island Hospital and Lifespan Medical Center, Providence, USA ${ }^{54}$ Warren Alpert Medical School of Brown University, Providence, USA ${ }^{55}$ Tumor Pathology Department, Maria Sklodowska-Curie Memorial Cancer Center, Gliwice, Poland ${ }^{56}$ Institute of Oncology, Gliwice Branch, Gliwice, Poland ${ }^{57}$ Department of Medicine, Vanderbilt University Medical Centre, Nashville, USA ${ }^{58}$ Vanderbilt Ingram Cancer Center, Nashville, USA ${ }^{59}$ Institute of Pathology, Charité Universitätsmedizin Berlin, Berlin, Germany ${ }^{60}$ VMscope $\mathrm{GmbH}$, Berlin, Germany ${ }^{61}$ Department of Pathology, Yale University School of Medicine, New Haven, USA ${ }^{62}$ Service de Biostatistique et d'Epidémiologie, Gustave Roussy, CESP, Inserm U1018, Université-Paris Sud, Université Paris-Saclay, Villejuif, France ${ }^{63} \mathrm{German}$ Breast Group $\mathrm{GmbH}$, NeuIsenburg, Germany ${ }^{64}$ Department of Pathology and Laboratory Medicine, University of British Columbia, Vancouver, Canada ${ }^{65}$ Trev \& Joyce Deeley Research Centre, British Columbia Cancer Agency, Victoria, British Columbia, Canada ${ }^{66}$ Department of Biochemistry and Microbiology, University of Victoria, Victoria, Canada ${ }^{67}$ Medical Genetics, University of British Columbia, Vancouver, British Columbia, Canada ${ }^{68}$ The Cancer Research Program, Garvan Institute of Medical Research, Darlinghurst, Australia ${ }^{69}$ Australian Clinical Labs, Bella Vista, Australia ${ }^{70}$ INSERM Unit U981, and Department of Medical Oncology, Gustave Roussy, Villejuif, France ${ }^{71}$ Faculté de Médecine, Université Paris Sud, Kremlin-Bicêtre, France ${ }^{72}$ Department of Surgical Oncology, Netherlands Cancer Institute, Amsterdam, The Netherlands ${ }^{73}$ Pathology Department, IIS-Fundacion Jimenez Diaz, UAM, Madrid, Spain ${ }^{74}$ Department of Pathology, University Hospital Leuven, Leuven, Belgium ${ }^{75}$ Department of Pathology and Laboratory Medicine, Weill Cornell Medical College, Doha, Qatar ${ }^{76}$ Department of Oncology, Montefiore Medical Centre, Albert Einstein College of Medicine, Bronx, USA ${ }^{77}$ Genetic Pathology Evaluation Centre, Department of Pathology and Laboratory Medicine, University of British Columbia, Vancouver, Canada ${ }^{78}$ Department of Pathology and Laboratory Medicine, University of Ottawa, Ottawa, Canada ${ }^{79}$ Laboratory of Pathology, Center for Cancer Research, National Cancer Institute, National Institutes of Health, Bethesda, MD, USA ${ }^{80}$ Department of Pathology, New York University Langone Medical Centre, New York, USA ${ }^{81}$ Directorate of Surgical Pathology, SA Pathology, Adelaide, Australia ${ }^{82}$ Discipline of Medicine, Adelaide University, Adelaide, Australia ${ }^{83}$ Pathology Department, Stanford University Medical Centre, Stanford, USA ${ }^{84}$ Division of Hematology-Oncology, Beth Israel Deaconess Medical Center, Boston, USA 85Department of Pathology, Netherlands Cancer Institute-Antoni van Leeuwenhoek, Amsterdam, The Netherlands ${ }^{86}$ Dana-Farber Cancer Institute, Boston, USA ${ }^{87}$ Pulmonary Pathology, New York University Center for Biospecimen Research and Development, New York University, New York, USA ${ }^{88}$ Division of Medical Oncology, Department of Medicine, University of Colorado Anschutz Medical Campus, Aurora, USA ${ }^{89}$ Department of Surgery, Oncology and Gastroenterology, University of Padova, Padua, Italy ${ }^{90}$ Medical Oncology 2, Veneto Institute of Oncology IOVIRCCS, Padua, Italy ${ }^{91}$ European Organisation for Research and Treatment of Cancer (EORTC) Headquarters, Brussels, Belgium ${ }^{92}$ Institute of Pathology, Medical 
University of Graz, Austria ${ }^{93}$ Pathology and Tissue Analytics, Roche Innovation Centre Munich, Penzberg, Germany ${ }^{94}$ Research Pathology, Genentech Inc., South San Francisco, USA ${ }^{95}$ Department of Pathology, Stanford University, Palo Alto, USA ${ }^{96}$ Translational Sciences, Medlmmune, Gaithersberg, USA ${ }^{97}$ Translational Medicine, Merck \& Co., Inc., Kenilworth, USA ${ }^{98}$ Department of Pathology, Memorial SloanKettering Cancer Center, New York, USA

\section{Acknowledgments}

Funding sources:

No specific funding was obtained for this project.

Roberto Salgado is supported by a grant from the Breast Cancer Research Foundation (BRCF).

Federico Rojo is supported by ISCiii/FEDER (CIBERONCO, PI15/00934).

Douglas B. Johnson receives funding from the NIH (NCI K23 CA204726).

We apologize to authors whose work could not be quoted due to space limitations.

\section{References}

1. Gajewski TF, Schreiber H, Fu YX. Innate and adaptive immune cells in the tumor microenvironment. Nat Immunol. 2013; 14:1014-1022. [PubMed: 24048123]

2. Coulie PG, Van den Eynde BJ, van der Bruggen P, et al. Tumour antigens recognized by $T$ lymphocytes: at the core of cancer immunotherapy. Nat Rev Cancer. 2014; 14:135-146. [PubMed: 24457417]

3. Fridman WH, Pages F, Sautes-Fridman C, et al. The immune contexture in human tumours: impact on clinical outcome. Nat Rev Cancer. 2012; 12:298-306. [PubMed: 22419253]

4. Galon J, Mlecnik B, Bindea G, et al. Towards the introduction of the 'Immunoscore' in the classification of malignant tumours. J Pathol. 2014; 232:199-209. [PubMed: 24122236]

5. Donnem T, Kilvaer TK, Andersen S, et al. Strategies for clinical implementation of TNMImmunoscore in resected nonsmall-cell lung cancer. Ann Oncol. 2016; 27:225-232. [PubMed: 26578726]

6. Masucci GV, Cesano A, Hawtin R, et al. Validation of biomarkers to predict response to immunotherapy in cancer: Volume I - pre-analytical and analytical validation. J Immunother Cancer. 2016; 4:76. [PubMed: 27895917]

7. Dobbin KK, Cesano A, Alvarez J, et al. Validation of biomarkers to predict response to immunotherapy in cancer: Volume II - clinical validation and regulatory considerations. J Immunother Cancer. 2016; 4:77. [PubMed: 27891226]

8. Simon RM, Paik S, Hayes DF. Use of archived specimens in evaluation of prognostic and predictive biomarkers. J Natl Cancer Inst. 2009; 101:1446-1452. [PubMed: 19815849]

9. Altman DG, McShane LM, Sauerbrei W, et al. Reporting Recommendations for Tumor Marker Prognostic Studies (REMARK): explanation and elaboration. PLoS Med. 2012; 9:e1001216. [PubMed: 22675273]

10. McShane LM, Altman DG, Sauerbrei W, et al. REporting recommendations for tumour MARKer prognostic studies (REMARK). Br J Cancer. 2005; 93:387-391. [PubMed: 16106245]

11. Crusz SM, Balkwill FR. Inflammation and cancer: advances and new agents. Nat Rev Clin Oncol. 2015; 12:584-596. [PubMed: 26122183]

12. Schreiber RD, Old LJ, Smyth MJ. Cancer immunoediting: integrating immunity's role in cancer suppression and promotion. Science. 2011; 331:1565-1570. [PubMed: 21436444] 
13. Dieu-Nosjean MC, Goc J, Giraldo NA, et al. Tertiary lymphoid structures in cancer and beyond. Trends Immunol. 2014; 35:571-580. [PubMed: 25443495]

14. Salgado R, Denkert C, Demaria S, et al. The evaluation of tumor-infiltrating lymphocytes (TILs) in breast cancer: recommendations by an International TILs Working Group 2014. Ann Oncol. 2015; 26:259-271. [PubMed: 25214542]

15. Schatton T, Scolyer RA, Thompson JF, et al. Tumor-infiltrating lymphocytes and their significance in melanoma prognosis. Methods Mol Biol. 2014; 1102:287-324. [PubMed: 24258985]

16. Klintrup K, Makinen JM, Kauppila S, et al. Inflammation and prognosis in colorectal cancer. Eur J Cancer. 2005; 41:2645-2654. [PubMed: 16239109]

17. Vayrynen JP, Vornanen JO, Sajanti S, et al. An improved image analysis method for cell counting lends credibility to the prognostic significance of T cells in colorectal cancer. Virchows Arch. 2012; 460:455-465. [PubMed: 22527018]

18. Pages F, Berger A, Camus M, et al. Effector Memory T Cells, Early Metastasis, and Survival in Colorectal Cancer. N Engl J Med. 2005; 353:2654-2666. [PubMed: 16371631]

19. Stack EC, Wang C, Roman KA, et al. Multiplexed immunohistochemistry, imaging, and quantitation: a review, with an assessment of Tyramide signal amplification, multispectral imaging and multiplex analysis. Methods. 2014; 70:46-58. [PubMed: 25242720]

20. Stack EC, Foukas PG, Lee PP. Multiplexed tissue biomarker imaging. J Immunother Cancer. 2016; 4:9. [PubMed: 26885371]

21. Carvajal-Hausdorf DE, Schalper KA, Neumeister VM, et al. Quantitative measurement of cancer tissue biomarkers in the lab and in the clinic. Lab Invest. 2015; 95:385-396. [PubMed: 25502176]

22. Cesano, A., Beecham, J., Webster, P., et al. Spatially-resolved, multiplexed digital characterization of protein distribution and abundance in FFPE tissue sections. [abstract]. Proceedings of the 107th Annual Meeting of the American Association for Cancer Research; 2016. Abstract nr 1371

23. Longuespee R, Fleron M, Pottier C, et al. Tissue proteomics for the next decade? Towards a molecular dimension in histology. OMICS. 2014; 18:539-552. [PubMed: 25105455]

24. Balluff B, Schone C, Hofler H, et al. MALDI imaging mass spectrometry for direct tissue analysis: technological advancements and recent applications. Histochem Cell Biol. 2011; 136:227-244. [PubMed: 21805154]

25. Young YK, Bolt AM, Ahn R, et al. Analyzing the Tumor Microenvironment by Flow Cytometry. Methods Mol Biol. 2016; 1458:95-110. [PubMed: 27581017]

26. Buisseret L, Garaud S, de Wind A, et al. Tumor-infiltrating lymphocyte composition, organization and PD-1/PD-L1 expression are linked in breast cancer. Oncoimmunology. 2017; 6:e1257452. [PubMed: 28197375]

27. Gentles AJ, Newman AM, Liu CL, et al. The prognostic landscape of genes and infiltrating immune cells across human cancers. Nat Med. 2015; 21:938-945. [PubMed: 26193342]

28. Pardoll DM. The blockade of immune checkpoints in cancer immunotherapy. Nat Rev Cancer. 2012; 12:252-264. [PubMed: 22437870]

29. Hodi FS, O'Day SJ, McDermott DF, et al. Improved survival with ipilimumab in patients with metastatic melanoma. N Engl J Med. 2010; 363:711-723. [PubMed: 20525992]

30. Robert C, Long GV, Brady B, et al. Nivolumab in previously untreated melanoma without BRAF mutation. N Engl J Med. 2015; 372:320-330. [PubMed: 25399552]

31. Rosenberg JE, Hoffman-Censits J, Powles T, et al. Atezolizumab in patients with locally advanced and metastatic urothelial carcinoma who have progressed following treatment with platinum-based chemotherapy: a single-arm, multicentre, phase 2 trial. The Lancet. 2016; 387:1909-1920.

32. Ansell SM, Lesokhin AM, Borrello I, et al. PD-1 blockade with nivolumab in relapsed or refractory Hodgkin's lymphoma. N Engl J Med. 2015; 372:311-319. [PubMed: 25482239]

33. Borghaei H, Paz-Ares L, Horn L, et al. Nivolumab versus Docetaxel in Advanced Nonsquamous Non-Small-Cell Lung Cancer. N Engl J Med. 2015; 373:1627-1639. [PubMed: 26412456]

34. Brahmer JR, Reckamp KL, Baas P, et al. Nivolumab versus Docetaxel in Advanced Squamous-Cell Non-Small-Cell Lung Cancer. N Engl J Med. 2015; 373:123-135. [PubMed: 26028407]

35. Garon EB, Rizvi NA, Hui R, et al. Pembrolizumab for the treatment of non-small-cell lung cancer. N Engl J Med. 2015; 372:2018-2028. [PubMed: 25891174] 
36. Motzer RJ, Escudier B, McDermott DF, et al. Nivolumab versus Everolimus in Advanced RenalCell Carcinoma. N Engl J Med. 2015; 373:1803-1813. [PubMed: 26406148]

37. Seiwert TY, Burtness B, Mehra R, et al. Safety and clinical activity of pembrolizumab for treatment of recurrent or metastatic squamous cell carcinoma of the head and neck (KEYNOTE-012): an open-label, multicentre, phase 1b trial. The Lancet Oncology. 2016; 17:956965. [PubMed: 27247226]

38. Topalian SL, Taube JM, Anders RA, et al. Mechanism-driven biomarkers to guide immune checkpoint blockade in cancer therapy. Nat Rev Cancer. 2016; 16:275-287. [PubMed: 27079802]

39. Garber K. Predictive biomarkers for checkpoints, first tests approved. Nat Biotechnol. 2015; 33:1217-1218. [PubMed: 26649999]

40. Kerr KM, Hirsch FR. Programmed Death Ligand-1 Immunohistochemistry: Friend or Foe? Arch Pathol Lab Med. 2016; 140:326-331. [PubMed: 26756647]

41. Tang H, Wang Y, Chlewicki LK, et al. Facilitating T Cell Infiltration in Tumor Microenvironment Overcomes Resistance to PD-L1 Blockade. Cancer Cell. 2016; 29:285-296. [PubMed: 26977880]

42. Ji RR, Chasalow SD, Wang L, et al. An immune-active tumor microenvironment favors clinical response to ipilimumab. Cancer Immunol Immunother. 2012; 61:1019-1031. [PubMed: 22146893]

43. Herbst RS, Soria JC, Kowanetz M, et al. Predictive correlates of response to the anti-PD-L1 antibody MPDL3280A in cancer patients. Nature. 2014; 515:563-567. [PubMed: 25428504]

44. Fehrenbacher L, Spira A, Ballinger M, et al. Atezolizumab versus docetaxel for patients with previously treated non-small-cell lung cancer (POPLAR): a multicentre, open-label, phase 2 randomised controlled trial. The Lancet. 2016; 387:1837-1846.

45. Leung SCY, Nielsen TO, Zabaglo L, et al. Analytical validation of a standardized scoring protocol for Ki67: phase 3 of an international multicenter collaboration. npj Breast Cancer. 2016; 2:16014. [PubMed: 28721378]

46. Moore OS, Foote FW. The relatively favorable prognosis of medullary carcinoma of the breast. Cancer. 1949; 2:635-640. [PubMed: 18144972]

47. Richardson WW. Medullary carcinoma of the breast: a distinctive tumour type with a relatively good prognosis following mastectomy. Br J Cancer. 1956; 10:415-423. [PubMed: 13396090]

48. Ridolfi RL, Rosen PP, Port A, et al. Medullary carcinoma of the breast: a clinicopathologic study with 10 year follow-up. Cancer. 1977; 40:1365-1385. [PubMed: 907958]

49. Bloom HJG, Richardson WW, Field JR. Host Resistance and Survival in Carcinoma of Breast: A Study of 104 Cases of Medullary Carcinoma in a Series of 1,411 Cases of Breast Cancer Followed for 20 years. BMJ. 1970; 3:181-188. [PubMed: 5448777]

50. Rapin V, Contesso G, Mouriesse H, et al. Medullary breast carcinoma: a reevaluation of 95 cases of breast cancer with inflammatory stroma. Cancer. 1988; 61:2503-2510. [PubMed: 2835145]

51. Stanton SE, Adams S, Disis ML. Variation in the Incidence and Magnitude of Tumor-Infiltrating Lymphocytes in Breast Cancer Subtypes: A Systematic Review. JAMA Oncol. 2016; 2:13541360. [PubMed: 27355489]

52. Savas P, Salgado R, Denkert C, et al. Clinical relevance of host immunity in breast cancer: from TILs to the clinic. Nat Rev Clin Oncol. 2016; 13:228-241. [PubMed: 26667975]

53. Mao Y, Qu Q, Chen X, et al. The Prognostic Value of Tumor-Infiltrating Lymphocytes in Breast Cancer: A Systematic Review and Meta-Analysis. PLoS One. 2016; 11:e0152500. [PubMed: 27073890]

54. Ibrahim EM, Al-Foheidi ME, Al-Mansour MM, et al. The Prognostic and Predicting Roles of Tumor-Infiltrating Lymphocytes in Breast Cancer: A Meta-Analysis. The Open Breast Cancer Journal. 2014; 6:9-19.

55. Yu X, Zhang Z, Wang Z, et al. Prognostic and predictive value of tumor-infiltrating lymphocytes in breast cancer: a systematic review and meta-analysis. Clin Transl Oncol. 2016; 18:497-506. [PubMed: 26459255]

56. Loi S, Drubay D, Adams S, et al. Abstract S1-03: Pooled individual patient data analysis of tumor infiltrating lymphocytes (TILs) in primary triple negative breast cancer (TNBC) treated with anthracycline-based chemotherapy. Cancer Res. 2016; 76:S1-03. 
57. Ibrahim EM, Al-Foheidi ME, Al-Mansour MM, et al. The prognostic value of tumor-infiltrating lymphocytes in triple-negative breast cancer: a meta-analysis. Breast Cancer Res Treat. 2014; 148:467-476. [PubMed: 25361613]

58. Loi S, Sirtaine N, Piette F, et al. Prognostic and predictive value of tumor-infiltrating lymphocytes in a phase III randomized adjuvant breast cancer trial in node-positive breast cancer comparing the addition of docetaxel to doxorubicin with doxorubicin-based chemotherapy: BIG 02-98. J Clin Oncol. 2013; 31:860-867. [PubMed: 23341518]

59. Loi S, Michiels S, Salgado R, et al. Tumor infiltrating lymphocytes are prognostic in triple negative breast cancer and predictive for trastuzumab benefit in early breast cancer: results from the FinHER trial. Ann Oncol. 2014; 25:1544-1550. [PubMed: 24608200]

60. Denkert C, Loibl S, Noske A, et al. Tumor-associated lymphocytes as an independent predictor of response to neoadjuvant chemotherapy in breast cancer. J Clin Oncol. 2010; 28:105-113. [PubMed: 19917869]

61. Denkert C, Von Minckwitz G, Brase JC, et al. Tumor-Infiltrating Lymphocytes and Response to Neoadjuvant Chemotherapy With or Without Carboplatin in Human Epidermal Growth Factor Receptor 2-Positive and Triple-Negative Primary Breast Cancers. J Clin Oncol. 2015; 33:983-991. [PubMed: 25534375]

62. Issa-Nummer Y, Darb-Esfahani S, Loibl S, et al. Prospective Validation of Immunological Infiltrate for Prediction of Response to Neoadjuvant Chemotherapy in HER2-Negative Breast Cancer - A Substudy of the Neoadjuvant GeparQuinto Trial. PLoS One. 2013; 8:e79775. [PubMed: 24312450]

63. West NR, Milne K, Truong PT, et al. Tumor-infiltrating lymphocytes predict response to anthracycline-based chemotherapy in estrogen receptor-negative breast cancer. Breast Cancer Res. 2011; 13:R126. [PubMed: 22151962]

64. Ali HR, Provenzano E, Dawson SJ, et al. Association between CD8+ T-cell infiltration and breast cancer survival in 12,439 patients. Ann Oncol. 2014; 25:1536-1543. [PubMed: 24915873]

65. Adams S, Gray RJ, Demaria S, et al. Prognostic value of tumor-infiltrating lymphocytes in triplenegative breast cancers from two phase III randomized adjuvant breast cancer trials: ECOG 2197 and ECOG 1199. J Clin Oncol. 2014; 32:2959-2966. [PubMed: 25071121]

66. Shou J, Zhang Z, Lai Y, et al. Worse outcome in breast cancer with higher tumor-infiltrating FOXP3+ Tregs : a systematic review and meta-analysis. BMC Cancer. 2016; 16:687. [PubMed: 27566250]

67. Jiang D, Gao Z, Cai Z, et al. Clinicopathological and prognostic significance of FOXP3+ tumor infiltrating lymphocytes in patients with breast cancer: a meta-analysis. BMC Cancer. 2015; 15:727. [PubMed: 26475790]

68. West NR, Kost SE, Martin SD, et al. Tumour-infiltrating FOXP3(+) lymphocytes are associated with cytotoxic immune responses and good clinical outcome in oestrogen receptor-negative breast cancer. Br J Cancer. 2013; 108:155-162. [PubMed: 23169287]

69. Bohling SD, Allison KH. Immunosuppressive regulatory T cells are associated with aggressive breast cancer phenotypes: a potential therapeutic target. Mod Pathol. 2008; 21:1527-1532. [PubMed: 18820666]

70. Liu S, Foulkes WD, Leung SCY, et al. Prognostic significance of FOXP3+ tumor-infiltrating lymphocytes in breast cancer depends on estrogen receptor and human epidermal growth factor receptor-2 expression status and concurrent cytotoxic T-cell infiltration. Breast Cancer Res. 2014; 16:432. [PubMed: 25193543]

71. Bates GJ, Fox SB, Han C, et al. Quantification of regulatory T cells enables the identification of high-risk breast cancer patients and those at risk of late relapse. J Clin Oncol. 2006; 24:53735380. [PubMed: 17135638]

72. Obeid M, Tesniere A, Ghiringhelli F, et al. Calreticulin exposure dictates the immunogenicity of cancer cell death. Nat Med. 2007; 13:54-61. [PubMed: 17187072]

73. Mattarollo SR, Loi S, Duret H, et al. Pivotal role of innate and adaptive immunity in anthracycline chemotherapy of established tumors. Cancer Res. 2011; 71:4809-4820. [PubMed: 21646474] 
74. Generali D, Bates G, Berruti A, et al. Immunomodulation of FOXP3+ regulatory T cells by the aromatase inhibitor letrozole in breast cancer patients. Clin Cancer Res. 2009; 15:1046-1051. [PubMed: 19188178]

75. Mao Y, Qu Q, Zhang Y, et al. The value of tumor infiltrating lymphocytes (TILs) for predicting response to neoadjuvant chemotherapy in breast cancer: a systematic review and meta-analysis. PLoS One. 2014; 9:e115103. [PubMed: 25501357]

76. Denkert C, von Minckwitz G, Darb-Esfahani S, et al. Abstract S1-09: Evaluation of tumorinfiltrating lymphocytes (TILs) as predictive and prognostic biomarker in different subtypes of breast cancer treated with neoadjuvant therapy - A metaanalysis of 3771 patients. Cancer Res. 2017; 77:S1-09.

77. Vinayak S, Gray RJ, Adams S, et al. Association of increased tumor-infiltrating lymphocytes (TILs) with immunomodulatory (IM) triple-negative breast cancer (TNBC) subtype and response to neoadjuvant platinum-based therapy in PrECOG0105. J Clin Oncol. 2014; 32:1000.

78. Dieci MV, Criscitiello C, Goubar A, et al. Prognostic value of tumor-infiltrating lymphocytes on residual disease after primary chemotherapy for triple-negative breast cancer: a retrospective multicenter study. Ann Oncol. 2014; 25:611-618. [PubMed: 24401929]

79. Garcia-Martinez E, Gil GL, Benito AC, et al. Tumor-infiltrating immune cell profiles and their change after neoadjuvant chemotherapy predict response and prognosis of breast cancer. Breast Cancer Res. 2014; 16:488. [PubMed: 25432519]

80. Workel HH, Komdeur FL, Wouters MC, et al. CD103 defines intraepithelial CD8+ PD1+ tumourinfiltrating lymphocytes of prognostic significance in endometrial adenocarcinoma. Eur J Cancer. 2016; 60:1-11. [PubMed: 27038842]

81. Wang ZQ, Milne K, Derocher H, et al. CD103 and Intratumoral Immune Response in Breast Cancer. Clin Cancer Res. 2016; 22:6290-6297. [PubMed: 27267849]

82. Mani NL, Schalper KA, Hatzis C, et al. Quantitative assessment of the spatial heterogeneity of tumor-infiltrating lymphocytes in breast cancer. Breast Cancer Res. 2016; 18:78. [PubMed: 27473061]

83. Nawaz S, Heindl A, Koelble K, et al. Beyond immune density: critical role of spatial heterogeneity in estrogen receptor-negative breast cancer. Mod Pathol. 2015; 28:766-777. [PubMed: 25720324]

84. Buisseret L, Desmedt C, Garaud S, et al. Reliability of tumor infiltrating lymphocyte and tertiary lymphoid structure assessment in human breast cancer. Mod Pathol. 2017 In press.

85. Pang JM, Gorringe KL, Fox SB. Ductal carcinoma in situ - update on risk assessment and management. Histopathology. 2016; 68:96-109. [PubMed: 26768032]

86. Van Bockstal M, Libbrecht L, Floris G, et al. The Baader-Meinhof phenomenon in ductal carcinoma in situ of the breast. Histopathology. 2016; 69:522-523. [PubMed: 27028714]

87. Thompson E, Taube JM, Elwood H, et al. The immune microenvironment of breast ductal carcinoma in situ. Mod Pathol. 2016; 29:249-258. [PubMed: 26769139]

88. Pruneri G, Lazzeroni M, Bagnardi V, et al. The prevalence and clinical relevance of tumorinfiltrating lymphocytes (TILs) in ductal carcinoma in situ of the breast. Ann Oncol. 2017; 28:321-328. [PubMed: 28426105]

89. Campbell MJ, Baehner F, O’Meara T, et al. Characterizing the immune microenvironment in highrisk ductal carcinoma in situ of the breast. Breast Cancer Res Treat. 2017; 161:17-28. [PubMed: 27785654]

90. Hussein MR, Hassan HI. Analysis of the mononuclear inflammatory cell infiltrate in the normal breast, benign proliferative breast disease, in situ and infiltrating ductal breast carcinomas: preliminary observations. J Clin Pathol. 2006; 59:972-977. [PubMed: 16935972]

91. Lal A, Chan L, Devries S, et al. FOXP3-positive regulatory T lymphocytes and epithelial FOXP3 expression in synchronous normal, ductal carcinoma in situ, and invasive cancer of the breast. Breast Cancer Res Treat. 2013; 139:381-390. [PubMed: 23712790]

92. Hiraoka N, Onozato K, Kosuge T, et al. Prevalence of FOXP3+ regulatory T cells increases during the progression of pancreatic ductal adenocarcinoma and its premalignant lesions. Clin Cancer Res. 2006; 12:5423-5434. [PubMed: 17000676] 
93. Cimino-Mathews A, Ye X, Meeker A, et al. Metastatic triple-negative breast cancers at first relapse have fewer tumor-infiltrating lymphocytes than their matched primary breast tumors: a pilot study. Hum Pathol. 2013; 44:2055-2063. [PubMed: 23701942]

94. Ogiya R, Niikura N, Kumaki N, et al. Comparison of tumor-infiltrating lymphocytes between primary and metastatic tumors in breast cancer patients. Cancer Sci. 2016; 107:1730-1735. [PubMed: 27727484]

95. Sobottka B, Pestalozzi B, Fink D, et al. Similar lymphocytic infiltration pattern in primary breast cancer and their corresponding distant metastases. Oncoimmunology. 2016; 5:e1153208. [PubMed: 27471624]

96. Baine MK, Turcu G, Zito CR, et al. Characterization of tumor infiltrating lymphocytes in paired primary and metastatic renal cell carcinoma specimens. Oncotarget. 2015; 6:24990-25002. [PubMed: 26317902]

97. Mansfield AS, Aubry MC, Moser JC, et al. Temporal and spatial discordance of programmed cell death-ligand 1 expression and lymphocyte tumor infiltration between paired primary lesions and brain metastases in lung cancer. Ann Oncol. 2016; 27:1953-1958. [PubMed: 27502709]

98. Ben-Avi R, TItzhaki O, Simansky D, et al. Metastatic Lung Lesions as a Preferred Resection Site for Immunotherapy With Tumor Infiltrating Lymphocytes. J Immunother. 2016; 39:218-222. [PubMed: 27163742]

99. Luen S, Salgado R, Fox SB, et al. Tumour-infiltrating lymphocytes in advanced HER2-positive breast cancer treated with pertuzumab or placebo in addition to trastuzumab and docetaxel: a retrospective analysis of the CLEOPATRA study. Lancet Oncology. 2017; 18:52-62. [PubMed: 27964843]

100. Mellor AL, Munn DH. Creating immune privilege: active local suppression that benefits friends, but protects foes. Nat Rev Immunol. 2008; 8:74-80. [PubMed: 18064049]

101. Bienkowski M, Preusser M. Prognostic role of tumour-infiltrating inflammatory cells in brain tumours: literature review. Curr Opin Neurol. 2015; 28:647-658. [PubMed: 26402405]

102. Berghoff AS, Fuchs E, Ricken G, et al. Density of tumor-infiltrating lymphocytes correlates with extent of brain edema and overall survival time in patients with brain metastases. Oncoimmunology. 2016; 5:e1057388. [PubMed: 26942067]

103. Mihm MC Jr, Clemente CG, Cascinelli N. Tumor Infiltrating Lymphocytes in Lymph Node Melanoma Metastases: A Histopathologic Prognostic Indicator and an Expression of Local Immune Response. Laboratory Investigation. 1996; 74:43-47. [PubMed: 8569196]

104. Cancer Genome Atlas N. Genomic Classification of Cutaneous Melanoma. Cell. 2015; 161:16811696. [PubMed: 26091043]

105. Kakavand H, Vilain RE, Wilmott JS, et al. Tumor PD-L1 expression, immune cell correlates and PD-1+ lymphocytes in sentinel lymph node melanoma metastases. Mod Pathol. 2015; 28:15351544. [PubMed: 26403784]

106. Bogunovic D, O’Neill DW, Belitskaya-Levy I, et al. Immune profile and mitotic index of metastatic melanoma lesions enhance clinical staging in predicting patient survival. Proc Natl Acad Sci U S A. 2009; 106:20429-20434. [PubMed: 19915147]

107. Schweiger T, Berghoff AS, Glogner C, et al. Tumor-infiltrating lymphocyte subsets and tertiary lymphoid structures in pulmonary metastases from colorectal cancer. Clin Exp Metastasis. 2016; 33:727-739. [PubMed: 27449756]

108. Halama N, Michel S, Kloor M, et al. Localization and density of immune cells in the invasive margin of human colorectal cancer liver metastases are prognostic for response to chemotherapy. Cancer Res. 2011; 71:5670-5677. [PubMed: 21846824]

109. Kwak Y, Koh J, Kim D-W, et al. Immunoscore encompassing CD3+ and CD8+ T cell densities in distant metastasis is a robust prognostic marker for advanced colorectal cancer. Oncotarget. 2016; 7:81778-81790. [PubMed: 27835889]

110. West NR, Panet-Raymond V, Truong PT, et al. Intratumoral Immune Responses Can Distinguish New Primary and True Recurrence Types of Ipsilateral Breast Tumor Recurrences (IBTR). Breast Cancer (Auckl). 2011; 5:105-115. [PubMed: 21695097] 
111. Dieci MV, Giaratano T, Miglietta F, et al. Abstract P2-05-20: Tumor infiltrating lymphocytes in recurrent HER2+ and triple negative breast cancer: Prognostic value according to tumor phenotype. Cancer Res. 2016; 77:P2-05-20.

112. Tumeh PC, Harview CL, Yearley JH, et al. PD-1 blockade induces responses by inhibiting adaptive immune resistance. Nature. 2014; 515:568-571. [PubMed: 25428505]

113. Lindeman NI, Cagle PT, Beasley MB, et al. Molecular Testing Guideline for Selection of Lung Cancer Patients for EGFR and ALK Tyrosine Kinase Inhibitors: Guideline from the College of American Pathologists, International Association for the Study of Lung Cancer, and Association for Molecular Pathology. Arch Pathol Lab Med. 2013; 137:828-860. [PubMed: 23551194]

114. Kilvaer TK, Paulsen EE, Khanehkenari MR, et al. The presence of intraepithelial CD45RO+ cells in resected lymph nodes with metastases from NSCLC patients is an independent predictor of disease-specific survival. Br J Cancer. 2016; 114:1145-1151. [PubMed: 27167450]

115. Psaila B, Lyden D. The metastatic niche: adapting the foreign soil. Nat Rev Cancer. 2009; 9:285293. [PubMed: 19308068]

116. Spitzer MH, Carmi Y, Reticker-Flynn NE, et al. Systemic Immunity Is Required for Effective Cancer Immunotherapy. Cell. 2017; 168:487-502. e415. [PubMed: 28111070]

117. Mlecnik B, Bindea G, Kirilovsky A, et al. The tumor microenvironment and Immunoscore are critical determinants of dissemination to distant metastasis. Science Translational Medicine. 2015; 8:327ra326.

118. Spranger S, Spaapen RM, Zha Y, et al. Up-Regulation of PD-L1, IDO, and Tregs in the Melanoma Tumor Microenvironment Is Driven by CD8+ T Cells. Sci Transl Med. 2013; 5:ra1 16.

119. Rooney MS, Shukla SA, Wu CJ, et al. Molecular and genetic properties of tumors associated with local immune cytolytic activity. Cell. 2015; 160:48-61. [PubMed: 25594174]

120. Rizvi N, Hellmann MD, Snyder A, et al. Mutational landscape determines sensitivity to PD-1 blockade in non-small cell lung cancer. Science. 2015; 348:124-128. [PubMed: 25765070]

121. Johnson DB, Frampton GM, Rioth MJ, et al. Targeted Next Generation Sequencing Identifies Markers of Response to PD-1 Blockade. Cancer Immunol Res. 2016; 4:959-967. [PubMed: 27671167]

122. Spranger S, Luke JJ, Bao R, et al. Density of immunogenic antigens does not explain the presence or absence of the T-cell-inflamed tumor microenvironment in melanoma. Proc Natl Acad Sci U S A. 2016; 113:E7759-E7768. [PubMed: 27837020]

123. Sweis RF, Spranger S, Bao R, et al. Molecular Drivers of the Non-T-cell-Inflamed Tumor Microenvironment in Urothelial Bladder Cancer. Cancer Immunol Res. 2016; 4:563-568. [PubMed: 27197067]

124. Danilova L, Wang H, Sunshine J, et al. Association of PD-1/PD-L axis expression with cytolytic activity, mutational load, and prognosis in melanoma and other solid tumors. Proc Natl Acad Sci U S A. 2016; 113:E7769-E7777. [PubMed: 27837027]

125. Smid M, Rodriguez-Gonzalez FG, Sieuwerts AM, et al. Breast cancer genome and transcriptome integration implicates specific mutational signatures with immune cell infiltration. Nat Commun. 2016; 7:12910. [PubMed: 27666519]

126. Spranger S, Bao R, Gajewski TF. Melanoma-intrinsic beta-catenin signalling prevents anti-tumour immunity. Nature. 2015; 523:231-235. [PubMed: 25970248]

127. Joyce JA, Fearon DT. T cell exclusion, immune privilege, and the tumor microenvironment. Science. 2015; 348:74-80. [PubMed: 25838376] 


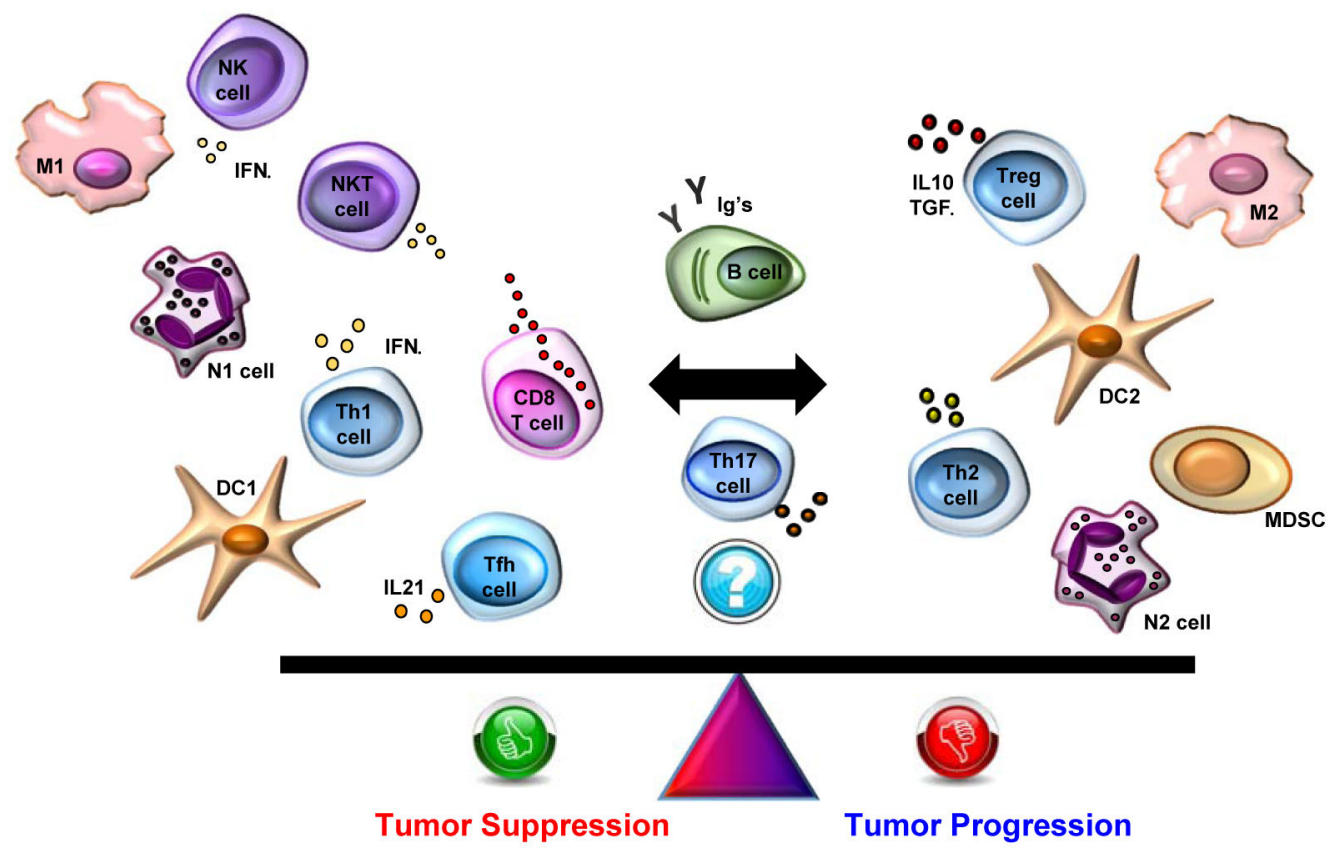

Figure 1.

The cellular constituents of the host immune response to tumors can control tumor growth or contribute to an immunosuppressive environment that promotes tumor progression. Breg, regulatory B cell; DC, dendritic cell; GC B cell, germinal center B cell; IFN $\gamma$, interferon gamma; Ig's, immunoglobulins; IL4, interleukin 4; IL10, interleukin 10; IL17, interleukin 17; IL21, interleukin 21; M, macrophage; MDSC, myeloid derived suppressor cell; N, neutrophil; NK, natural killer cell; NKT, natural killer T cell; Tfh, follicular B helper T cells; $\mathrm{Th}$, helper $\mathrm{CD}^{+}{ }^{+} \mathrm{T}$ cell; TGF $\beta$, transforming growth factor beta; TILs, tumor-infiltrating lymphocytes; Treg, regulatory $\mathrm{T}$ cell. 


\section{Standardized approach for TILs evaluation in solid tumors}

\section{Step 1: Select tumor area}

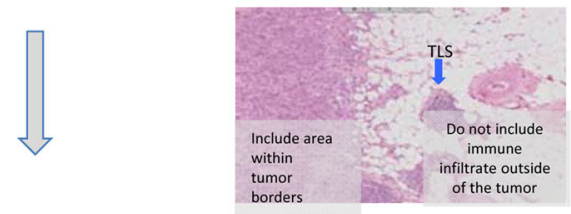

Step 2: Define stromal and intra-tumoral areas

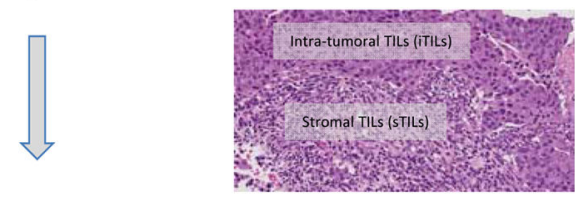

Step 3: Scan at low magnification

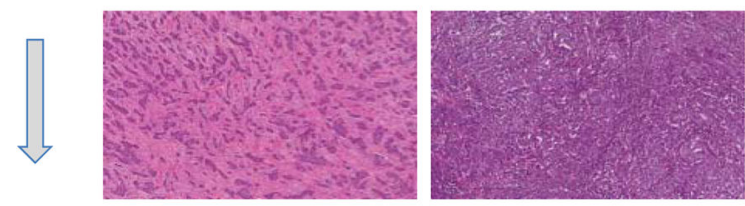

Step 4: Determine type of inflammatory infiltrate

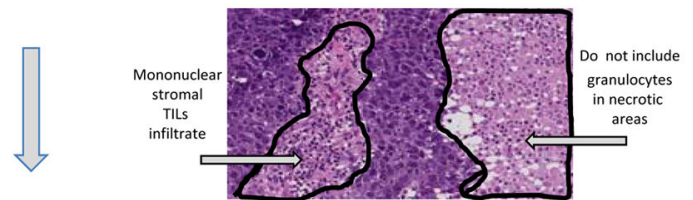

Step 5: Assess the percentage TILs

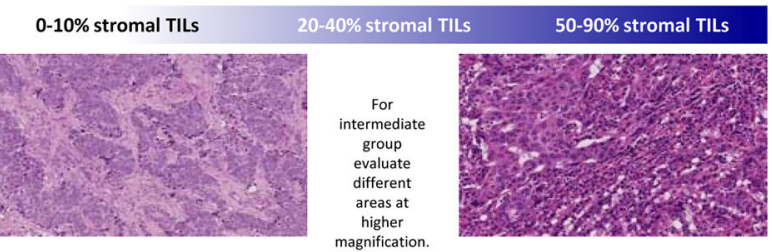

Figure 2.

Guidelines for a standardized approach to TILs evaluation in solid tumors. Modified from Salgado et al [14] with permission from Oxford University Press on behalf of the European Society for Medical Oncology. 


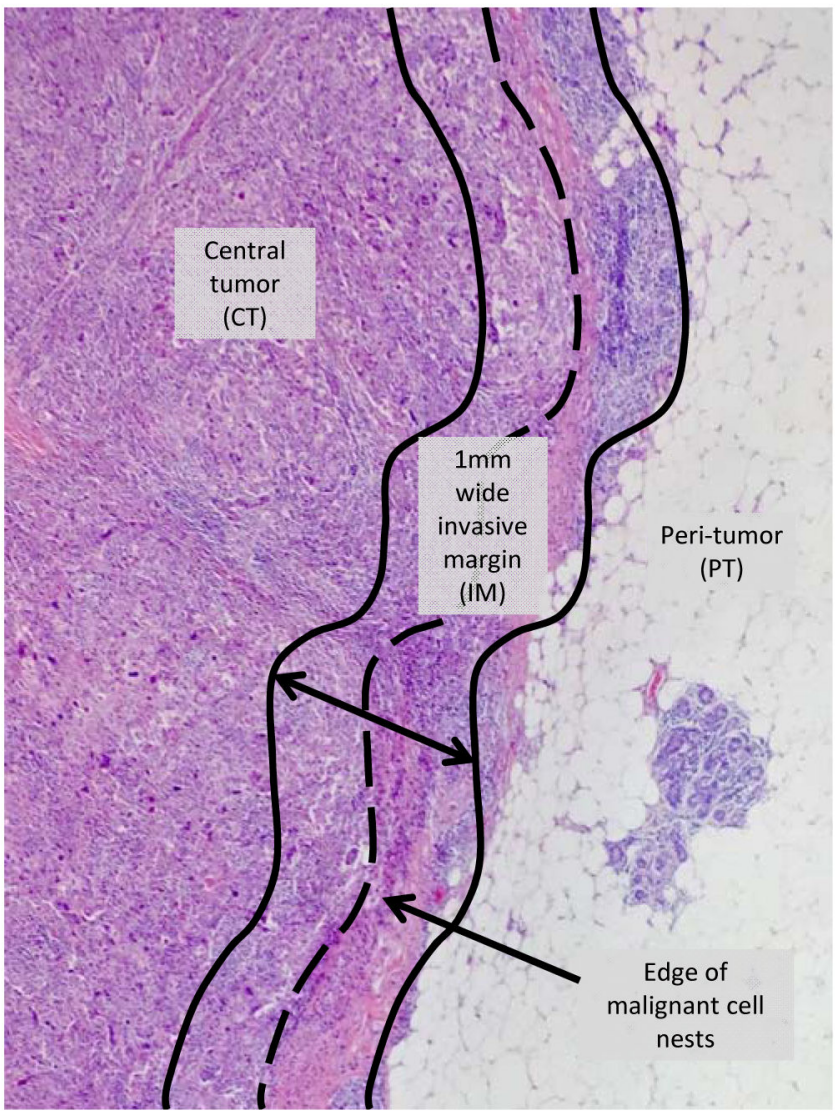

Figure 3.

The "invasive margin" (IM) is defined as the region centered on the border separating the host tissue from the malignant nests, with an extent of $1 \mathrm{~mm}$. "Central tumor" (CT) corresponds to all the tissue inside the IM, and "peri-tumor" (PT) to tissue outside of the IM. 

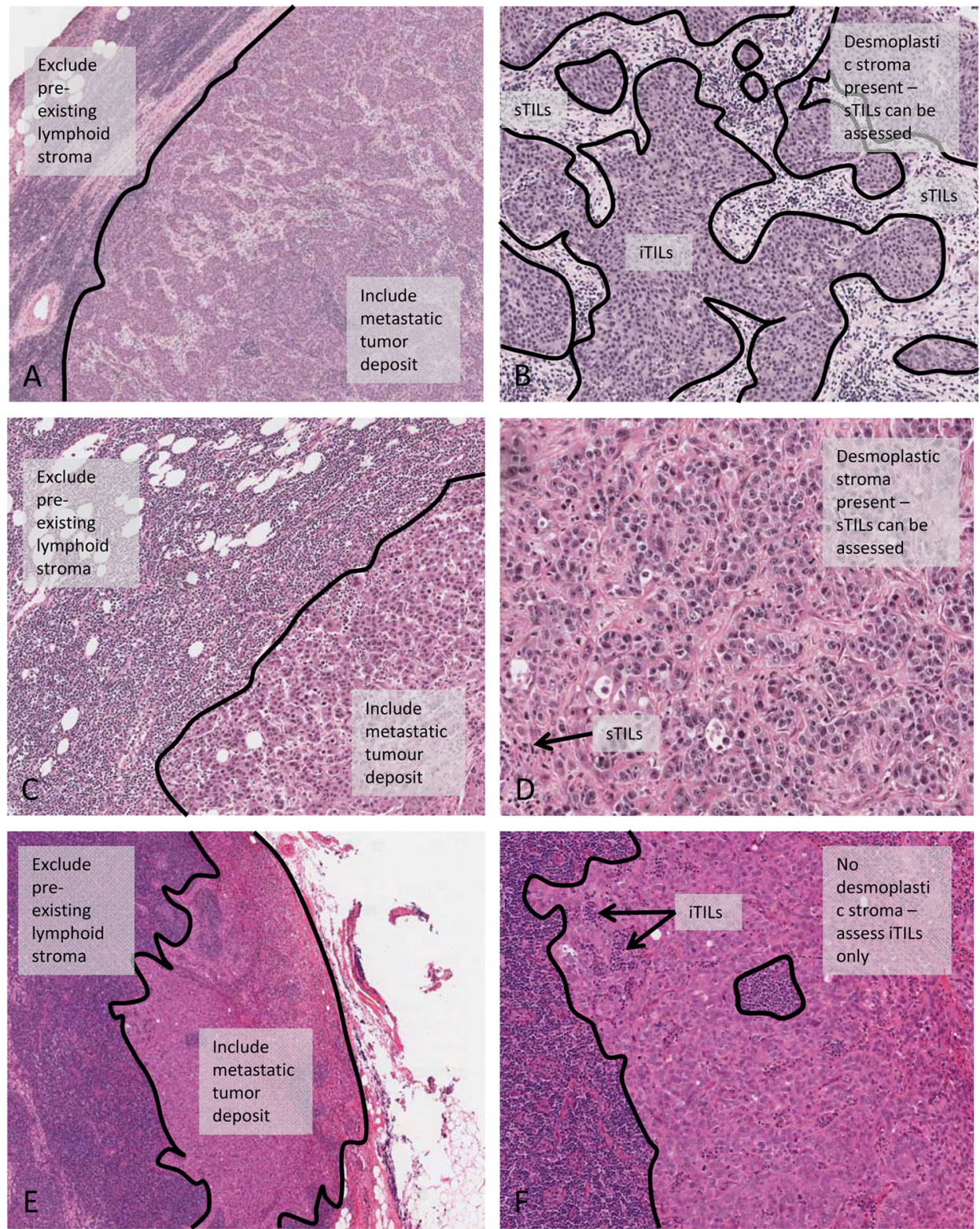

Figure 4.

Assessing TILs in metastatic tumor deposits in lymph nodes. Cases showing a desmoplastic stroma can be scored as for the primary lesion, i.e. sTILs may be scored within this reactive stroma (Panels A-D). Panels A and B show an example of a metastatic tumor deposit with clearly distinguishable border between pre-existing lymphoid tissue and tumoral sTILs.

Panels C and D show an example with very few sTILs in the stroma of the tumor deposit. In cases without a desmoplastic stroma, TILs are scored only within tumor nests (Panels E and F), i.e. iTILs only. The pre-existing lymphoid stroma is excluded from the evaluation. 


\section{Table 1}

Proposed guidelines for the assessment of tumor-infiltrating lymphocytes (TILs) in solid tumors: recommendations by an International Immuno-Onoclogy Biomarker Working Group.

1. TILs should be reported separately for the stromal compartment (= \% stromal TILs) and the tumor cell compartment ( $=\%$ intra-tumoral TILs). The reasons are 1) in many tumors the TIL density in both compartments is different, and 2) if the TILs are evaluated simply per tumor area, the density and growth pattern of tumor cells (= a non-immune parameter) will affect the TIL count. The denominator used to determine the \% stromal TILs is the area of stromal tissue (i.e. area occupied by mononuclear inflammatory cells over total stromal area), not the number of stromal cells (i.e. fraction of total stromal nuclei that represent mononuclear inflammatory cell nuclei). Similarly, for intra-tumoral TILs the tumor cell area is the denominator. In some tumor types, e.g. breast cancer, it might be decided to evaluate only the stromal TILs.

2. TILs should be evaluated within the borders of the invasive tumor, including both "central tumor" and "invasive margin". These areas may be reported separately when required.

3. The "invasive margin" is defined as a $1 \mathrm{~mm}$ region centered on the border separating the malignant cell nests from the host tissue. The "central tumor" represents the remaining tumor area.

4. Exclude TILs at a distance outside of the tumor borders. TILs immediately adjacent to the invasive margin, i.e. "peri-tumoral TILs", may be evaluated when required.

5. Exclude TILs in tumor zones with crush artifacts, necrosis and regressive hyalinization, as well as in previous biopsy sites.

6. All mononuclear cells (including lymphocytes and plasma cells) should be scored, but polymorphonuclear leukocytes (neutrophils) should be excluded.

7. One section (4-5 $\mu \mathrm{m}$, magnification 200-400x) per patient can be considered to be sufficient for practical purposes. However, assessing additional sections for each case whenever possible and reporting the number of sections reviewed per case specifically in the manuscript is recommended since the extent of heterogeneity for different tumor types is unknown.

8. Full sections are preferred over biopsies whenever possible. Cores can be used in the pre-therapeutic neoadjuvant setting; currently no validated methodology has been developed to score TILs after neo-adjuvant treatment.

9. A full assessment of average TILs in the tumor area (central tumor and invasive margin) should be used. Do not focus on hotspots.

10. TILs should be assessed as a continuous variable, as this may provide more biologically relevant information and allow more accurate statistical analyses. However, in daily practice most pathologists will report discrete estimates, for example $13.5 \%$ will be rounded to $15 \%$. Pathologists should report their scores in as much detail as the pathologist feels comfortable with.

11. For assessment of percentage values, the dissociated growth pattern of lymphocytes needs to be taken into account. The percentage of stromal TILs is a semi-quantitative parameter for this assessment, for example, $80 \%$ stromal TILs means that $80 \%$ of the stromal area shows a dense mononuclear infiltrate. Lymphocytes typically do not form solid cellular aggregates, therefore the designation "100\% stromal TILs" would still allow some empty tissue space between the individual lymphocytes.

12. No formal recommendation for a clinically relevant TIL threshold(s) can be given at this stage. A valid methodology is currently more important than issues of thresholds for clinical use, which will be determined once a solid methodology is in place. 


\title{
Table 2
}

Additional points for attention when assessing TILs in different tumor types.

\author{
Invasive breast carcinoma \\ - $\quad$ Refer to Salgado et al [14]. Consensus guidelines are reproduced in Table 3.

\section{Ductal carcinoma in situ and other pre-malignant lesions} \\ - $\quad$ Refer to Pruneri et al 2016 [88]. \\ - Stromal area is defined as the specialized stroma surrounding the ducts involved by in-situ carcinoma, or when this is not clear, \\ an area surrounding the ducts within 2 high power fields (approximately $1 \mathrm{~mm}$ ). \\ - Any type of circumferential infiltrate should be taken into account, including minimal, partial, subtotal and total circumferential \\ TILs. \\ - Exclude TILs that are in continuity between the invasive tumor and the in-situ lesions with no clear distinction as to whether \\ these are TILs associated with the invasive or in situ component.

\section{Melanoma} \\ - Currently only iTILs are scored in the clinical setting. sTILs and/or peri-tumoral TILs may be reported separately in research \\ settings. \\ - Only the vertical growth phase of the primary tumor is evaluated. \\ - $\quad$ Further research may determine what \%iTILs corresponds to the traditional categorization of brisk/non-brisk/absent.
}

Colorectal carcinoma

- Separately reporting invasive margin and central tumor TILs is recommended. Invasive margin TILs appear to have the most prognostic significance in this setting.

\section{Upper gastrointestinal tract carcinomas}

- $\quad$ Early evidence supports evaluating only sTILs in gastric carcinoma, due to a lack of prognostic significance of iTILs. This finding requires further validation.

- There is insufficient data on pancreatic ductal adenocarcinoma and hepatocellular carcinoma to make specific recommendations.

Non-small cell lung carcinoma

- $\quad$ sTILs and iTILs should be separately reported in the research setting at present. Insufficient evidence is available to support evaluating only sTILs over a combined assessment.

- Do not include areas with pure intra-alveolar tumor spread (aerogenic spread) or with pure lepidic growth (no desmoplastic reaction).

- $\quad$ Do not include alveolar macrophages.

\section{Ovarian carcinoma}

- Both the central tumor and invasive margin should be included, but it is not currently recommended to report these regions separately.

- $\quad$ iTILs and sTILs should be separately reported at present, as both compartments appear to have prognostic relevance. Further research may determine the relative importance of the different compartments.

- Include TILs in the stroma pertaining to fibrovascular cores of papillary structures.

- Include the invasive margin of superficial peritoneal or ovarian deposits.

Head and neck squamous cell carcinoma

- $\quad$ Care should be taken to exclude pre-existing lymphoid stroma from the assessment in oropharyngeal (i. e. tonsillar and base of tongue) tumors. If a desmoplastic stroma is present, sTILs can be scored in this reactive stroma (as for lymph node metastases).

Genitourinary carcinomas 
- Separate reporting of iTILs and sTILs is recommended - this is important in the context of immune checkpoint inhibitor therapy with atezolizumab in urothelial carcinoma, where the PD-L1 "immune cell" score is derived from the sTILs score.

- Care should be taken to avoid areas of diathermy/coagulation artifact, a common finding in bladder tumor specimens.

- Non-invasive papillary structures are not currently included in the assessment.

- There is insufficient data on prostate carcinoma and renal cell carcinoma to make specific recommendations.

Primary brain tumors

- $\quad$ No evidence based recommendations on the optimal method for TIL quantification can be made at present.

- Immunohistochemistry may be required to clearly identify TILs and distinguish immune cells from pre-existing or neoplastic glial and neuronal cells.

- Consideration should be given to separately reporting TILs in the central tumor, perivascular areas, perinecrotic areas and the invasive margin.

Metastatic tumor deposits

- In metastatic deposits within lymph nodes, if a desmoplastic stroma is present, sTILs and iTILs can be scored as for the primary lesion. If a desmoplastic stroma is not present, focus only on iTILs. Exclude the pre-existing lymphoid stroma.

- Other sites are evaluated as for the primary lesion.

- Future research should focus on the relative clinical utility of evaluating TILs in the primary tumor or in the most recent tumor tissue available. 
Table 3

The evaluation of tumor-infiltrating lymphocytes (TILs) in breast cancer: recommendations by an International TILs Working Group 2014

\author{
Reproduced from Salgado et al [14] with permission from Oxford University Press on behalf of the European \\ Society for Medical Oncology.
}

1. TILs should be reported for the stromal compartment (=\% stromal TILs). The denominator used to determine the $\%$ stromal TILs is the area of stromal tissue (i.e. area occupied by mononuclear inflammatory cells over total intra-tumoral stromal area), not the number of stromal cells (i.e. fraction of total stromal nuclei that represent mononuclear inflammatory cell nuclei)

2. TILs should be evaluated within the borders of the invasive tumor

3. Exclude TILs outside of the tumor border and around DCIS and normal lobules.

4. Exclude TILs in tumor zones with crush artifacts, necrosis, regressive hyalinization as well as in the previous core biopsy site.

5. All mononuclear cells (including lymphocytes and plasma cells) should be scored, but polymorphonuclear leukocytes are excluded.

6. One section $(4-5 \mu \mathrm{m}$, magnification $\times 200-400)$ per patient is currently considered to be sufficient.

7. Full sections are preferred over biopsies whenever possible. Cores can be used in the pretherapeutic neoadjuvant setting; currently no validated methodology has been developed to score TILs after neoadjuvant treatment.

8. A full assessment of average TILs in the tumor area by the pathologist should be used. Do not focus on hotspots.

9. The working group's consensus is that TILs may provide more biological relevant information when scored as a continuous variable, since this will allow more accurate statistical analyses, which can later be categorized around different thresholds. However, in daily practice, most pathologists will rarely report for example $13.5 \%$ and will round up to the nearest $5 \%-10 \%$, in this example thus $15 \%$. Pathologist should report their scores in as much detail as the pathologist feels comfortable with.

10. TILs should be assessed as a continuous parameter. The percentage of stromal TILs is a semi-quantitative parameter for this assessment, for example, $80 \%$ stromal TILs means that $80 \%$ of the stromal area shows a dense mononuclear infiltrate. For assessment of percentage values, the dissociated growth pattern of lymphocytes needs to be taken into account. Lymphocytes typically do not form solid cellular aggregates; therefore, the designation ' $100 \%$ stromal TILs' would still allow some empty tissue space between the individual lymphocytes.

11. No formal recommendation for a clinically relevant TIL threshold(s) can be given at this stage. The consensus was that a valid methodology is currently more important than issues of thresholds for clinical use, which will be determined once a solid methodology is in place.

Lymphocyte predominant breast cancer can be used as a descriptive term for tumors that contain 'more lymphocytes than tumor cells'.

However, the thresholds vary between $50 \%$ and $60 \%$ stromal lymphocytes. 